\title{
Eigenfunction Expansions and Scattering Theory for Dirac Operators
}

By

\author{
Osanobu YAMADA*
}

\section{§0. Introduction}

In the present paper we are concerned with the Dirac operator

$$
L=-i \sum_{j=1}^{3} \alpha_{j} \frac{\partial}{\partial x_{j}}+\beta+Q(x)
$$

in $\mathbb{R}^{3}$, where $\alpha_{j}$ and $\beta$ are the so-called Dirac matrices and $Q(x)$ is a Hermitian symmetric potential decaying at infinity with like $|x|^{-1-h}$, $h>0$. More detailed assumptions will be mentioned in $\$ 2$.

Eigenfunction expansions and scattering problems for Dirac operators have been investigated by many authors. Titchmarsh [14] verifies the completeness of eigenfunctions, assuming that $Q(x)$ is a spherically symmetric scalar function. Eckardt [4], Evans [5] and Thompson [13] treat the potential $Q(x)=0\left(|x|^{-2-h}\right)(h>0)$, stimulated by Ikebe's work [7] on eigenfunction expansions for Schrödinger operators. Mochizuki [10] discusses the perturbation of the absolutely continuous spectrum under the main assumption $|Q(x)|+\sum_{j=1}^{3}\left|\frac{\partial Q}{\partial x_{j}}\right|=0\left(|x|^{-2-h}\right)$. Prosser [11] proves the existence of wave operators supposing mainly $Q(x)$ $=0\left(|x|^{-1-h}\right)$, and shows the unitarity of the scattering operator for a class of potentials with compact support. Eckardt [3] assures the existence of wave operators under the condition $|Q(x)|(1+|x|)^{-1 / 2+\varepsilon}$ $\in L^{2}\left(\mathbf{R}^{3}\right), \varepsilon>0$.

In this paper we deal with the Hermitian symmetric potential $Q(x)$ $=0\left(|x|^{-1-h}\right)$ and consider expansion formulae, wave and scattering

Communicated by S. Matsuura, March 27, 1975.

* Department of Mathematics, Ritsumeikan University, Kyoto, Japan. 
operators. It will be found that the scattering operator is unitary under a condition weaker than Prosser's. Our eigenfunctions are determined as kernels of linear operators

$$
\begin{aligned}
& A_{v}\left(\tau_{v} \sqrt{r^{2}+1} \pm 0 i, r\right) h \\
& \quad=\left(I-R^{ \pm}\left(\tau_{v} \sqrt{r^{2}+1}\right) Q\right) \int_{S} \Phi_{v}^{0}(x, r \omega) h(\omega) d \omega, \quad r>0 \\
& \quad\left(v=p, n, \tau_{p}=+1, \tau_{n}=-1 \text { and } S \text { is the unit sphere }\right),
\end{aligned}
$$

where $\Phi_{v}^{0}$ is the eigenfunction of the unperturbed operator $(Q(x) \equiv 0)$ and $R^{ \pm}(\lambda)$ is the "boundary value" of the resolvent $R(z)$ of the perturbed operator as $z \rightarrow \lambda \pm 0 i$. The above idea is suggested by the works of Agmon [1], [2] on selfadjoint elliptic operators. The principle of limiting absorption and Sobolev's imbedding theorems are important tools for us to study the operator $A_{v}(\lambda \pm 0 i, r)$. The principle of limiting absorption is a method to investigate the behavior of the resolvent $R(z)$ near the real line. A work of the author [15] for the principle of limiting absorption will be needed in our discussion.

We shall outline the contents of this paper. In $\S 1$ the eigenfunctions and the expansion theorem for the unperturbed operator are introduced in terms of Fourier transforms. The assumptions on the potential $Q(x)$ and the principle of limiting absorption will be explained in $\S 2$. In $\S 3$ we shall define an operator

$$
A_{v}(z, r) h=(I-R(z) Q) \int_{S} \Phi_{v}^{0}(x, r \omega) h(\omega) d \omega, \quad r>0
$$

and research the behavior of $A_{v}(z, r)$ and its adjoint operator as $z$ tends to the real line. Our eigenfunctions and the eigenfunction expansions for the perturbed operator are found in $\S 4$, Theorems 4.4 and 4.5. In $\S 5$ we prove the orthogonality of the eigenfunctions. In $\S 6$ we define the stationary wave operator and show that it coincides with the time-dependent wave operator and that the scattering operator is unitary. 


\section{§1. The Unperturbed Operator}

We shall consider in this section the eigenfunction expansions of the unperturbed Dirac operator

$$
L_{0}=-i \sum_{j=1}^{3} \alpha_{j} \frac{\partial}{\partial x_{j}}+\beta, \quad i=\sqrt{-1},
$$

where $\alpha_{j}$ and $\beta$ are $4 \times 4$ constant Hermitian symmetric matrices satisfying the anti-commutation relations

$$
\alpha_{j} \alpha_{k}+\alpha_{k} \alpha_{j}=2 \delta_{j k} I, \quad j, k=1,2,3,4,
$$

$\left(\alpha_{4}=\beta\right.$ and $I$ is the unit matrix, $\delta_{j k}$ is Kronecker's delta). A complete set of matrix-valued eigenfunctions is given by

$$
\Phi_{p}^{0}(x, \xi)=\frac{1}{2}\left(I+\frac{\hat{L}_{0}(\xi)}{\sqrt{|\xi|^{2}+1}}\right) e^{i<x, \xi>}
$$

and

$$
\Phi_{n}^{0}(x, \xi)=\frac{1}{2}\left(I-\frac{\hat{L}_{0}(\xi)}{\sqrt{|\xi|^{2}+1}}\right) e^{i<x, \xi>}
$$

for $\xi \in \mathbb{R}^{3}$, where $\hat{L}_{0}(\xi)=\sum_{j=1}^{3} \xi_{j} \alpha_{j}+\beta$ and $<\cdot, \cdot>$ denotes the inner product of $\mathbb{R}^{3}$. The function $\Phi_{v}^{0}(x, \xi)$ satisfies

$$
L_{0} \Phi_{v}^{0}(x, \xi)=\tau_{v} \sqrt{|\xi|^{2}+1} \Phi_{v}^{0}(x, \xi) \quad(v=p, n),
$$

where $\tau_{p}=+1$ and $\tau_{n}=-1$, since

$$
\left(\hat{L}_{0}(\xi)\right)^{2}=\left(|\xi|^{2}+1\right) I
$$

as is easily checked by using the anti-commutation relations. Let us set

$$
\psi_{v}(\xi)=\frac{1}{2}\left(I+\tau_{v} \frac{\hat{L}_{0}(\xi)}{\sqrt{|\xi|^{2}+1}}\right) .
$$

$\psi_{\nu}(\xi)$ are symmetric matrices and fulfill

$$
\begin{gathered}
\psi_{v}(\xi)^{2}=\psi_{v}(\xi), \quad v=p, n \\
\psi_{p}(\xi)+\psi_{n}(\xi)=I \quad \text { and } \psi_{p}(\xi) \psi_{n}(\xi)=\psi_{n}(\xi) \psi_{p}(\xi)=0
\end{gathered}
$$


We consider Dirac operators in the Hilbert space $\mathscr{L}^{2}=\left(L^{2}\left(\mathbb{R}^{3}\right)\right)^{4}$, a class of all $\mathbb{C}^{4}$-valued functions $u(x)=\left(u_{1}(x), u_{2}(x), u_{3}(x), u_{4}(x)\right)$ such that

$$
\|u\|_{\mathscr{f}^{2}}^{2}=\int_{\mathbb{R}^{3}}|u(x)|^{2} d x<+\infty
$$

with the inner product

$$
(u, v)=\int_{\mathbb{R}^{3}}<u(x), v(x)>d x,
$$

where $|u(x)|^{2}=\sum_{j=1}^{4}\left|u_{j}(x)\right|^{2}$ and $\left\langle u(x), v(x)>=\sum_{j=1}^{4} u_{j}(x) \overline{v_{j}(x)}\right.$. The above fact (1.2) implies

$$
\mathscr{L}^{2}=\psi_{p} \mathscr{L}^{2}+\psi_{n} \mathscr{L}^{2}
$$

that is, $\mathscr{L}^{2}$ is the orthogonal sum of $\psi_{p} \mathscr{L}^{2}$ and $\psi_{n} \mathscr{L}^{2}$, where $\psi_{v} \mathscr{L}^{2}$ denotes the image of $\mathscr{L}^{2}$ under the multiplicative operator by $\psi_{v}(\cdot)$. Now we introduce

$$
\left(Z_{v}^{0} f\right)(\xi)=\hat{f}_{v}^{0}(\xi)=(2 \pi)^{-3 / 2} \text { l.i.m. } \int \Phi_{v}^{0}(-x, \xi) f(x) d x
$$

for $f \in \mathscr{L}^{2}$, where 1.i.m. $\int \ldots d x$ means the limit in the mean of $\int_{|x| \leqq R} \ldots d x$ as $R \rightarrow \infty$. Using the Fourier transform of $f(x)$

$$
\hat{f}(\xi)=(2 \pi)^{-3 / 2} \text { l.i.m. } \int e^{-i<\xi, x>} f(x) d x,
$$

we see immediately that $\hat{f}_{v}^{0}(\xi)=\psi_{v}(\xi) \hat{f}(\xi)$, which shows that $Z_{v}^{0}$ maps $\mathscr{L}^{2}$-functions into $\psi_{v} \mathscr{L}^{2}$. Since the Fourier transform maps $\mathscr{L}^{2}$ onto $\mathscr{L}^{2}$, the range of $Z_{v}^{0}$ is identical with $\psi_{v} \mathscr{L}^{2}$.

In consequence of the Fourier inversion formula and (1.2) we have

$$
\begin{aligned}
f(x) & =(2 \pi)^{-3 / 2} 1 \cdot \mathrm{i} \cdot \mathrm{m} \cdot \int e^{i<x, \xi>\hat{f}(\xi) d \xi} \\
& =(2 \pi)^{-3 / 2} \text { l.i.m. } \int\left(\Phi_{p}^{0}(x, \xi)+\Phi_{n}^{0}(x, \xi)\right) \hat{f}(\xi) d \xi \\
& =(2 \pi)^{-3 / 2} \text { li.m. } \int\left(\Phi_{p}^{0}(x, \xi) \hat{f}_{p}^{0}(\xi)+\Phi_{n}^{0}(x, \xi) \hat{f}_{n}^{0}(\xi)\right) d \xi
\end{aligned}
$$


for $f \in \mathscr{L}^{2}$ and

$$
\begin{aligned}
\left(L_{0} f\right)(x)= & (2 \pi)^{-3 / 2} 1 \cdot \mathrm{i} \cdot \mathrm{m} \cdot \int e^{i<x, \xi>} \hat{L}_{0}(\xi) \hat{f}(\xi) d \xi \\
= & (2 \pi)^{-3 / 2} 1 \cdot \mathrm{i} \cdot \mathrm{m} \cdot \int\left(\sqrt{|\xi|^{2}+1} \Phi_{p}^{0}(x, \xi)\right. \\
& \left.-\sqrt{|\xi|^{2}+1} \Phi_{n}^{0}(x, \xi)\right) \hat{f}(\xi) d \xi \\
= & (2 \pi)^{-3 / 2} 1 \cdot \mathrm{i} \cdot \mathrm{m} \cdot \int\left(\sqrt{|\xi|^{2}+1} \Phi_{p}^{0}(x, \xi) \hat{f}_{p}^{0}(\xi)\right. \\
& \left.-\sqrt{|\xi|^{2}+1} \Phi_{n}^{0}(x, \xi) \hat{f}_{n}^{0}(\xi)\right) d \xi
\end{aligned}
$$

for $f \in \mathscr{H}^{1}\left(\mathbf{R}^{3}\right)$, where $\mathscr{H}^{1}\left(\mathbf{R}^{3}\right)$ is the Sobolev space of all $\mathscr{L}^{2}$-functions with the first derivatives in $\mathscr{L}^{2}$. Thus the eigenfunction expansion problems for the unperturbed operator is completely solved.

\section{§2. The Assumption on the Potential $Q(x)$ and the Principle of Limiting Absorption}

Throughout this paper we assume the following condition (A) on the potential $Q(x)$ :

(A) $Q(x)=\left(q_{i j}(x)\right)$ is a $4 \times 4$ Hermitian matrix-valued function with continuously differentiable components $q_{i j}(x)$ except at a finite number of singularities, and there exist positive numbers $h, R_{0}$ and $p>3$, such that

$$
|Q(x)| \leqq \frac{\text { const. }}{(1+|x|)^{1+h}} \quad\left(|x| \geqq R_{0}\right)
$$

$$
\sum_{j=1}^{3}\left|\frac{\partial Q}{\partial x_{j}}\right| \leqq \text { const . } \quad\left(|x| \geqq R_{0}\right)
$$

and

$$
\int_{|x| \leqq R_{0}}|Q(x)|^{p}<+\infty
$$

where

$$
|Q(x)|=\left(\sum_{i, j=1}^{4}\left|q_{i j}(x)\right|^{2}\right)^{1 / 2}
$$


In our previous paper ([15], Propositions 2.3, 2.4 and Corollary 4.2) we obtain

Proposition 2.1. $L_{0}$ and $L=L_{0}+Q(x)$ have unique self-adjoint realizations $H_{0}$ and $H=H_{0}+Q$, respectively, with the domain $D(H)$ $=D\left(H_{0}\right)=\mathscr{H}^{1}\left(\mathbb{R}^{3}\right)$, where $Q$ is the maximal multiplication operator by $Q(x)$. The essential spectrum of $H_{0}$ and $H$ coincides with intervals $(-\infty,-1]$ and $[+1,+\infty)$, while $(-1,+1)$ is contained in the resolvent set of $H_{0}$. The set $(-\infty,-1) \cup(+1,+\infty)$ is the absolutely continuous spectrum of $H_{0}$ and $H$.

Before we proceed, we introduce some functional spaces. For $m$ a non-negative integer, $H^{m}(\Omega)$ denotes the Sobolev space of all $\mathbb{L}^{2}(\Omega)$ functions satisfying

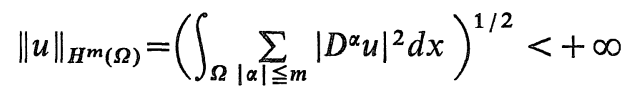

( $\Omega$ is an open set). For $s$ a real number and $m$ a non-negative integer we put

$$
\begin{aligned}
L_{s}^{2} & =\left\{u(x) ;\|u\|_{L_{s}^{2}}=\left(\int_{\mathbb{R}^{3}}(1+|x|)^{2 s}|u(x)|^{2} d x\right)^{1 / 2}<+\infty\right\}, \\
H_{s}^{m} & =\left\{u(x) ;\|u\|_{H_{s}^{m}}=\left(\int_{\mathbb{R}^{3}}(1+|x|)^{2 s} \sum_{|\alpha| \leqq m}\left|D^{\alpha} u\right|^{2} d x\right)^{1 / 2}<+\infty\right\} .
\end{aligned}
$$

We denote by $\mathscr{L}^{2}(\Omega), \mathscr{H}^{m}(\Omega), \mathscr{L}_{s}^{2}$, and $\mathscr{H}_{s}^{m}$ the product spaces $\left(\mathbb{L}^{2}(\Omega)\right)^{4}$, $\left(H^{m}(\Omega)\right)^{4},\left(L_{s}^{2}\right)^{4}$ and $\left(H_{s}^{m}\right)^{4}$, respectively. When $\Omega=\mathbb{R}^{3}$, the symbol $(\Omega)$ will be often omitted.

We shall now explain the principle of limiting absorption. Let $R(z)$ $=(H-z)^{-1}$ be the resolvent of $H$ for every complex number $z$ such that $\operatorname{Im} z \neq 0(\operatorname{Im} z(\operatorname{Re} z)$ is the imaginary (real) part of $z)$. Then $R(z)$ is a bounded operator on $\mathscr{L}^{2}$ to $\mathscr{H}^{1}$. According to [15], Theorem 4.1, for any $s>1 / 2$ and $-\infty<a<b<-1$ or $+1<a<b<+\infty$ there exists a positive constant $C=C(a, b, s)$ such that

$$
\|R(z) f\|_{\mathscr{L}_{-s}^{1}} \leqq C\|f\|_{\mathscr{L}_{s}^{2}}
$$

for all $f \in \mathscr{L}_{s}^{2}$ and $z \in J_{+}(a, b)\left(J_{-}(a, b)\right)$, where $J_{+}(a, b)\left(J_{-}(a, b)\right)$ is the 
set of all complex numbers $z$ such that $a \leqq \operatorname{Re} z \leqq b$ and $0<\operatorname{Im} z \leqq 1$ $(-1 \leqq \operatorname{Im} z<0)$. The following proposition is also shown in [15], Corollary 4.1 .

Proposition 2.2 (the principle of limiting absorption). For every real $\lambda,|\lambda|>1$, there exist bounded operators $R^{+}(\lambda)$ and $R^{-}(\lambda)$ on $\mathscr{L}_{s}^{2}$ to $\mathscr{H}_{-s}^{1}(s>1 / 2)$ such that

$$
\underset{z \rightarrow \lambda+0 i}{\mathrm{~s}-\lim _{\mathrm{im}}} R(z) f=R^{+}(\lambda) f, \underset{z \rightarrow \lambda-0 i}{\mathrm{~s}-\lim _{z \rightarrow}} R(z) f=R^{-}(\lambda) f
$$

in $\mathscr{H}_{-s}^{1}$ for any $f \in \mathscr{L}_{s}^{2}$. For every $f \in \mathscr{L}_{s}^{2}, R(z) f$ is strongly continuous in $\mathscr{H}_{-s}^{1}$ with respect to $\left.z \in \overline{J_{+}(a, b)} \overline{\left(J_{-}(a, b)\right.}\right)$ (the closure of $J_{+}(a, b)$ $\left(J_{-}(a, b)\right)$ with the boundary value $R^{+}(\lambda) f\left(R^{-}(\lambda) f\right)$.

The following property will be frequently used hereafter.

Proposition 2.3. Let $1 / 2<t<(1+h) / 2$ ( $h$ is the positive number in the condition (a.1)). Then the multiplicative operator $Q$ on $\mathscr{H}_{-t}^{1}$ to $\mathscr{L}_{t}^{2}$ is compact.

Proof. Take an arbitrary bounded sequence $\left\{f_{j}\right\}$ in $\mathscr{H}_{-}^{1}{ }_{t}$. In view of Rellich's theorem $\left\{f_{j}\right\}$ has a subsequence converging locally in $\mathscr{L}^{2}$, which we rewrite $\left\{f_{j}\right\}$. We separate the integral

$$
\int_{\mathbf{R}^{3}}(1+|x|)^{2 t}\left|Q f_{j}-Q f_{k}\right|^{2} d x
$$

into three integrals $\int_{|x| \leqq R_{0}} \ldots d x, \int_{R_{0} \leqq|x| \leqq R} \ldots d x$ and $\int_{|x| \geqq R} \ldots d x$. To the first integral we apply Corollary 2.1 in [15], which asserts that for any $\varepsilon>0$ there exists a positive constant $C_{\varepsilon}$ such that

$$
\int_{|x| \leqq R_{0}}|Q u|^{2} d x \leqq \varepsilon \int_{|x| \leqq R_{0}+1}\left|L_{0} u\right|^{2} d x+C_{\varepsilon} \int_{|x| \leqq R_{0}+1}|u(x)|^{2} d x
$$

for all $u \in \mathscr{H}^{1}\left(B_{R_{0}+1}\right)$, where $B_{R}$ denotes the ball of radius $R$ about the origin. So we estimate the first integral

$$
\begin{aligned}
\int_{|x| \leqq R} \ldots d x & \leqq C_{1}\left(\varepsilon \int_{|x| \leqq R_{0}+1}\left|L_{0} f_{j}-L_{0} f_{k}\right|^{2} d x+C_{\varepsilon} \int_{|x| \leqq R_{0}+1}\left|f_{j}-f_{k}\right|^{2} d x\right) \\
& \leqq \varepsilon C_{2}+C_{1} C_{\varepsilon} \int_{|x| \leqq R_{0}+1}\left|f_{j}-f_{k}\right|^{2} d x,
\end{aligned}
$$


where the constants $C_{1}, C_{2}$ are independent of $j$ and $k$. For the second integral we obtain

$$
\int_{R_{0} \leqq|x| \leqq R} \ldots d x \leqq C_{3}(R) \int_{R_{0} \leqq|x| \leqq R}\left|f_{j}-f_{k}\right|^{2} d x .
$$

The condition (a.1) yields for the last integral

$$
\begin{aligned}
\int_{|x| \geqq R} \ldots d x & \leqq C_{4} \int_{|x| \geqq R}(1+|x|)^{2 t-2-2 h}\left|f_{j}-f_{k}\right|^{2} d x \\
& \leqq C_{5}(1+R)^{4 t-2-2 h} \longrightarrow 0 \quad(R \rightarrow \infty),
\end{aligned}
$$

where $C_{4}$ and $C_{5}$ do not depend on $j, k$ and $R$. These estimates show the compactness of $Q$.

Q.E.D.

\section{§3. Auxiliary Lemmas}

The greater part of this section is devoted to studying a linear operator $A_{v}(z, r)$ defined by

$$
A_{v}(z, r) h=(I-R(z) Q) \int_{S} \Phi_{v}^{0}(x, r \omega) h(\omega) d \omega, \quad v=p, n
$$

for $r>0$ and $h \in \mathscr{L}^{2}(S)$, where $S$ is the unit sphere about the origin. Our aim is to give an expression to $R(z) f$ and $\widehat{R(z) f}$ for $f \in \mathscr{L}_{t}^{2}(t>1 / 2)$ by means of $A_{v}(z, r)$.

The following proposition is well-known as Sobolev's imbedding theorem (see, e.g., Sobolev [12], p. 85).

Proposition 3.1. (1) For any $s>1 / 2,0<\theta<s-1 / 2(\theta \leqq 1)$ and $0<a$ $<b$ there exist positive constants $C_{s}$ and $C_{s, \theta}=C_{s, \theta}(a, b)$ such that

$$
\|f(r \cdot)\|_{L^{2}(s)} \leqq C_{s}\|f\|_{H^{s}}
$$

and

$$
\left\|f\left(r_{1} \cdot\right)-f\left(r_{2} \cdot\right)\right\|_{L^{2}(S)} \leqq C_{s, \theta}\left|r_{1}-r_{2}\right|^{\theta}\|f\|_{H^{s}}
$$

for $f \in H^{s}$ and $a \leqq r, r_{1}, r_{2} \leqq b$, where $H^{s}$ denotes the class of all temperate distribution whose Fourier transforms are $L_{s}^{2}$-functions, normed 
by $\|u\|_{H^{s}}=\|\hat{u}\|_{L_{s}^{2}}$.

(2) All $H^{2}\left(\mathbb{R}^{3}\right)$-functions are bounded and uniformly Hörder continuous i.e.,

$$
|f(x)| \leqq \text { const. }\|f\|_{H^{2}}
$$

and

$$
\left|f\left(x_{1}\right)-f\left(x_{2}\right)\right| \leqq C_{\theta}\left|x_{1}-x_{2}\right|^{\theta}\|f\|_{H^{2}}
$$

for $f \in H^{2}, 0<\theta<1 / 2$ and $x_{1}, x_{2} \in \mathbb{R}^{3}$.

The following proposition may be a dual statement of Proposition 3.1, (1).

Proposition 3.2. For any $s>1 / 2,0<\theta<s-1 / 2(\theta \leqq 1)$ and $0<a<b$ there exist positive constants $C_{s}$ and $C_{s, \theta}=C_{s, \theta}(a, b)$ such that

$$
\begin{aligned}
& \left\|\int_{S} e^{i<x, \omega>} h(\omega) d \omega\right\|_{L_{-s}^{2}} \leqq C_{s}\|h\|_{L^{2}(S)} \\
& \left\|\int_{S}\left(e^{i<x, r_{1} \omega>}-e^{i<x, r_{2} \omega>}\right) h(\omega) d \omega\right\|_{L_{-s}^{2}} \leqq C_{s, \theta}\left|r_{1}-r_{2}\right|^{\theta}\|h\|_{L^{2}(S)}
\end{aligned}
$$

for $h \in L^{2}(S), a \leqq r_{1}, r_{2} \leqq b$.

Proof. Let $g$ be an arbitrary $C_{0}^{\infty}$-function. Then we have

$$
\int_{\mathbb{R}^{3}} \overline{g(x)} d x \int_{S} e^{i<x, \omega>} h(\omega) d \omega=(h, \hat{g})_{L^{2}(S)}
$$

and

$$
\begin{aligned}
\left|(h, \hat{g})_{L^{2}(S)}\right| & \leqq h\left\|_{L^{2}(S)}\right\| \hat{g} \|_{L^{2}(S)} \\
& \leqq C_{s}\|h\|_{L^{2}(S)}\|\hat{g}\|_{H^{s}}=C_{s}\|h\|_{L^{2}(S)}\|g\|_{L_{s}^{2}}
\end{aligned}
$$

by virtue of Proposition 3.1. Since $C_{0}^{\infty}$ is dense in $L_{s}^{2}$, and $L_{-s}^{2}$ is the dual space of $L_{s}^{2}$, the above inequality shows (3.2). A similar argument can be applied to get (3.3). (The above proof has been suggested by Prof. Ikebe.)

Q.E.D.

The above proposition is made use of to estimate the integral 
$\int_{S} \Phi_{v}^{0}(x, r \omega) h(\omega) d \omega$ in $(3.1)$

Lemma 3.3. Let $1 / 2<t<(1+h) / 2$. Then every $A_{v}(z, r)$ is a bounded operator on $\mathscr{L}^{2}(S)$ to $\mathscr{H}_{-}^{1}$, satisfying

$$
\left(L_{0}+Q(x)-z\right) A_{v}(z, r) h=\left(\tau_{v} \sqrt{r^{2}+1}-z\right) \int_{S} \Phi_{v}^{0}(x, r \omega) h(\omega) d \omega
$$

for $h \in \mathscr{L}^{2}(S)$. For each $J_{+}(a, b)\left(J_{-}(a, b)\right), 0<\theta<t-1 / 2$ and $0<\alpha<\beta$ there exist positive constants $C_{1}=C_{1}(t)$ and $C_{2}=C_{2}(t, \theta)$ such that

$$
\begin{gathered}
\left\|A_{v}(z, r) h\right\|_{\mathscr{C}^{1} t} \leqq C_{1}\|h\|_{\mathscr{L}^{2}(S)}, \\
\left\|A_{v}\left(z, r_{1}\right) h-A_{v}\left(z, r_{2}\right)\right\| h\left\|_{\mathscr{C}_{-t}^{1}} \leqq C_{2}\left|r_{1}-r_{2}\right|^{\theta}\right\| h \|_{\mathscr{L}^{2}(S)}
\end{gathered}
$$

for $h \in \mathscr{L}^{2}(S), \alpha \leqq r, r_{1}, r_{2} \leqq \beta, z \in J_{+}(a, b)\left(J_{-}(a, b)\right)$.

Proof. In the first place we shall prove

$$
\left\|\int_{S} \Phi_{v}^{0}(x, r \omega) h(\omega) d \omega\right\|_{\mathscr{C}_{-t}^{1}} \leqq C_{3}(t)\|h\|_{\mathscr{L}^{2}(S)},
$$

where the constant $C_{3}(t)$ is independent of $r, \alpha \leqq r \leqq \beta$. The estimate for the part involving no derivatives in the left-hand side of (3.7) follows directly from Proposition 3.2. For the first derivatives we see that

$$
\frac{\partial}{\partial x_{j}} \int_{S} \Phi_{v}^{0}(x, r \omega) h(\omega) d \omega=\int_{S} \Phi_{v}^{0}(x, r \omega)\left(i r \omega_{j}\right) h(\omega) d \omega
$$

Applying Proposition 3.2 again to (3.8) gives (3.7). Next we proceed to show

$$
\left\|R(z) Q(x) \int_{S} \Phi_{v}^{0}(x, r \omega) h(\omega) d \omega\right\|_{\mathscr{C}_{-t}^{1}} \leqq C_{4}(t)\|h\|_{\mathscr{L}^{2}(S)}
$$

$C_{4}(t)$ being a positive constant independent of $r, \alpha \leqq r \leqq \beta$, and of $z$ $\in J_{ \pm}(a, b)$. The above inequality is obtained in view of Proposition 2.3 and (2.1). Thus (3.5) is proved, and (3.6) is also proved by similar estimates. Finally, in order to verify (3.4), we need only to take account 
of (1.1) and (3.8). Then we get

$$
\begin{aligned}
\left(L_{0}+Q(x)-z\right) A_{v}(z, r) h \\
\quad=\left(L_{0}+Q(x)-z\right)(I-R(z) Q) \int_{S} \Phi_{v}^{0}(x, r \omega) h(\omega) d \omega \\
\quad=\left(L_{0}-z\right) \int_{S} \Phi_{v}^{0}(x, r \omega) h(\omega) d \omega \\
\quad=\left(\tau_{v} \sqrt{r^{2}+1}-z\right) \int_{S} \Phi_{v}^{0}(x, r \omega) h(\omega) d \omega .
\end{aligned}
$$

Corollary 3.4. $1 / 2<t<(1+h) / 2$. Let

$$
A_{v}(\lambda \pm 0 i, r) h=\left(I-R^{ \pm}(\lambda) Q\right) \int_{S} \Phi_{v}^{0}(x, r \omega) h(\omega) d \omega
$$

for $h \in \mathscr{L}^{2}(S)$ and for every real $\lambda$ such that $|\lambda|>1$. Then we have

$$
\left\|A_{v}(z, r) h-A_{v}(\lambda \pm 0 i, r) h\right\|_{\mathscr{C}_{-t}^{1}} \longrightarrow 0 \quad(z \rightarrow \lambda \pm 0 i)
$$

for $h \in \mathscr{L}^{2}(S)$. (3.4), (3.5) and (3.6) hold for $z \in \overline{J_{+}(a, b)}\left(\overline{J_{-}(a, b)}\right)$.

Proof. As shown in the above lemma, $\int_{S} \Phi_{v}^{0}(x, r \omega) h(\omega) d \omega$ belongs to $\mathscr{H}_{-t}^{1}$ for $r>0$, and, consequently, $Q(x) \int_{S} \Phi_{v}^{0}(x, r \omega) h(\omega) d \omega$ belongs to $\mathscr{L}_{t}^{2}$ for $r>0$ as a result of Proposition 2.3. Proposition 2.2 enables us to obtain

$$
\begin{gathered}
R(z) Q(\cdot) \int_{S} \Phi_{v}^{0}(\cdot, r \omega) h(\omega) d \omega \\
\longrightarrow R^{ \pm}(\lambda) Q(\cdot) \int_{S} \Phi_{v}^{0}(\cdot, r \omega) h(\omega) d \omega \quad \text { in } \quad \mathscr{H}_{-t}^{1}
\end{gathered}
$$

as $z \rightarrow \lambda \pm 0 i$. Since the estimates (3.5) and (3.6) are independent of $z \in J_{+}(a, b)\left(J_{-}(a, b)\right)$, they are also valid on the closure $\overline{J_{+}(a, b)}\left(\overline{J_{-}(a, b)}\right)$ by taking the limit as $z \rightarrow \lambda+0 i(\lambda-0 i)$.

Let $C_{0}^{\infty}\left(\mathbf{R}^{3}-\{0\}\right)$ be the family of all $C^{\infty}$-functions with compact support in $\mathbb{R}^{3}-\{0\}$, and $\mathscr{C}_{0}^{\infty}=\left(C_{0}^{\infty}\left(\mathbb{R}^{3}\right)\right)^{4}, \mathscr{C}_{0}^{\infty}\left(\mathbb{R}^{3}-\{0\}\right)=\left(C_{0}^{\infty}\left(\mathbb{R}^{3}-\{0\}\right)\right)^{4}$.

From now on, we assume $1 / 2<t<(1+h) / 2$. 
Lemma 3.5. Let $\operatorname{Im} z \neq 0$ and

$$
V_{v}(z) f=(2 \pi)^{-3 / 2} \int_{0}^{\infty} \frac{A_{v}(z, r) \hat{f}(r \cdot)}{\tau_{v} \sqrt{r^{2}+1-z}} r^{2} d r \quad(v=p, n)
$$

for $f$ such that $\hat{f}(\xi)=\hat{f}(r \omega) \in \mathscr{C}_{0}^{\infty}\left(R^{3}-\{0\}\right)$, where and in what follows the integrals are taken in Bochner's sense (cf. e.g., Hille and Phillips [6], chapter III). Then we have

$$
R(z) f=V_{p}(z) f+V_{n}(z) f
$$

Proof. The integral (3.10) makes sense, for $A_{v}(z, r) \hat{f}(r \cdot)$ is a $\mathscr{H}{ }_{-}^{1}$-valued continuous function of $r>0$ with compact support in $(0,+\infty)$ (the continuity is ensured by Lemma 3.3 and the smoothness of $\hat{f}$ ). For any $\psi \in \mathscr{C}_{0}^{\infty}$ we have

$$
\int_{\mathbf{R}^{3}}<V_{p}(z) f+V_{n}(z) f,(L-\bar{z}) \psi>d x
$$

$$
=(2 \pi)^{-3 / 2} \int_{0}^{+\infty} r^{2} d r \int_{\mathbb{R}^{3}}<\left(\frac{A_{p}(z, r)}{\sqrt{r^{2}+1}-z}-\frac{A_{n}(z, r)}{\sqrt{r^{2}+1}+z}\right) \hat{f}(r \cdot),(L-\bar{z}) \psi>d x
$$

by use of the following Proposition 3.6, (2). (1.2) and (3.4) yield

$$
\begin{aligned}
(3.11) & =(2 \pi)^{-3 / 2} \int_{0}^{+\infty} r^{2} d r \int_{\mathbb{R}^{3}}<\int_{S}\left(\Phi_{p}^{0}(x, r \omega)+\Phi_{n}^{0}(x, r \omega)\right) \hat{f}(r \omega) d \omega, \psi(x)>d x \\
& =(2 \pi)^{-3 / 2} \int_{0}^{+\infty} r^{2} d r \int_{\mathbb{R}^{3}}<\int_{S} e^{i<x, r \omega>} \hat{f}(r \omega) d \omega, \psi(x)>d x \\
& =\int_{\mathbb{R}^{3}}<f(x), \psi(x)>d x .
\end{aligned}
$$

Therefore we have

$$
(L-z)\left(V_{p}(z) f+V_{n}(z) f\right)=f .
$$

In order to complete the proof we have to show

$$
V_{p}(z) f+V_{n}(z) f \in \mathscr{H}^{1} .
$$

To this end we arrange (3.12) as

$$
\left(L_{0}-z\right)\left(V_{p}(z) f+V_{n}(z) f\right)=f-Q\left(V_{p}(z) f+V_{n}(z) f\right) .
$$


The right-hand side of (3.13), say $g$, belongs to $\mathscr{L}^{2}$ because of Proposition 2.3. Taking the Fourier transforms of both sides of (3.13), we obtain

$$
\widehat{V_{p}(z) f}+\widehat{V_{n}(z) f}=\left(\hat{L}_{0}(\check{\xi})-z\right)^{-1} \hat{g}=\frac{\hat{L}_{0}(\xi)+z}{|\xi|^{2}+1-z^{2}} \hat{g},
$$

which implies $V_{p}(z)+V_{n}(z) f \in \mathscr{H}^{1}$, because $\hat{g} \in \mathscr{L}^{2}$. Thus we may replace $L$ with $H$ in (3.12).

Q.E.D.

We summarize several properties of Bochner integrals used in the paper (for the proof see Hille and Phillips [6], p. 73-p. 83).

Proposition 3.6. Let $(S, \mathscr{F}, \mu)$ be a measure space and $X$ a Banach space, $X^{*}$ the dual space. We denote by $\int_{F} x(s) d \mu$ the Bochner integral for an $X$-valued integrable function $x(s)$ defined on $F \in \mathscr{F}$.

(1) (Pettis) If $X$ is separable, then strong and weak measurability are equivalent notions.

(2) For any $x^{*} \in X^{*}$,

$$
<x^{*}, \int_{F} x(s) d \mu>=\int_{F}<x^{*}, x(s)>d \mu,
$$

where $\langle\cdot, \cdot\rangle$ denotes the dual pairing of $X^{*}$ and $X$.

(3) Let $T$ be a closed operator in $X$. If $x(s) \in D(T)$ (the domain of $T)$ and $x(s), T x(s)$ are integrable on $F$, then $\int_{F} x(s) d \mu \in D(T)$ and

$$
T\left[\int_{F} x(s) d \mu\right]=\int_{F} T x(s) d \mu .
$$

We shall consider the adjoint operator $A_{v}^{*}(z, r)$, mapping the dual space $\mathscr{H}_{t}^{-1}$ of $\mathscr{H}_{-t}^{1}$ to $\mathscr{L}^{2}(S) . \mathscr{H}_{t}^{-1}$ can be regarded as the completion of $\mathscr{C}_{0}^{\infty}$ with the norm

$$
\|f\|_{\mathscr{P}_{t}^{-1}}=\sup _{\|g\|_{\mathscr{C}^{1}-t}^{-1}}\left|\int_{\mathbf{R}^{3}}<g(x), f(x)>d x\right| .
$$

Noticing that $\mathscr{H}_{-t}^{1} \subset \mathscr{L}_{-t}^{2}$ and $\|u\|_{\mathscr{L}_{-t}^{2}} \leqq\|u\|_{\mathscr{C}_{-t}^{1}}$ for $u \in \mathscr{H}_{-t}^{1}$, we have $\mathscr{L}_{t}^{2} \subset \mathscr{H}_{t}^{-1}$ and 


$$
\|v\|_{\mathscr{P}_{t}^{-1}} \leqq\|v\|_{\mathscr{L}_{t}^{2}}
$$

for $v \in \mathscr{L}_{t}^{2}$. The above inequality (3.14) shows that the restriction of $A_{v}^{*}(z, r)$ on $\mathscr{L}_{t}^{2}$ is continuous.

Lemma 3.7. For every $f \in \mathscr{L}_{t}^{2}$

$$
\begin{aligned}
A_{v}^{*}(z, r) f & =(2 \pi)^{3 / 2}\left\{\left(Z_{v}^{0} f\right)(r \cdot)-\left(Z_{v}^{0} Q R(\bar{z}) f\right)(r \cdot)\right\} \\
& =(2 \pi)^{3 / 2} \psi_{v}(r \cdot)(\hat{f}(r \cdot)-\widehat{Q R(\bar{z}) f}(r \cdot)) .
\end{aligned}
$$

Proof. Let us take an arbitrary $f \in \mathscr{L}^{2}$ with compact support. Then we obtain

$$
\begin{aligned}
\int_{\mathbf{R}^{3}} & <A_{v}(z, r) h, f(x)>d x \\
= & \int_{\mathbf{R}^{3}}<\int_{S} \Phi_{v}^{0}(x, r \omega) h(\omega) d \omega, f(x)-Q(x) R(\bar{z}) f>d x \\
= & \int_{S}<h(\omega), \int_{\mathbb{R}^{3}} \Phi_{v}^{0}(-x, r \omega)(f(x)-Q(x) R(\bar{z}) f) d x>d \omega
\end{aligned}
$$

for $h \in \mathscr{L}^{2}(S)$, where we used Fubini's theorem by virtue of the absolute integrability

$$
\begin{array}{r}
\int_{\mathbf{R}^{3}} \int_{S}\left|\Phi_{v}^{0}(x, r \omega)\right||h(\omega)|(|f(x)|+|Q(x) R(\bar{z}) f|) d \omega d x \\
\leqq \text { const. }\|h\|_{\mathscr{L}^{2}(S)} \int_{\mathbf{R}^{3}}(|f(x)|+|Q(x) R(\bar{z}) f|) d x<+\infty
\end{array}
$$

(the summability of $Q(x) R(\bar{z}) f$ will be shown below). Thus (3.15) holds good for $f \in \mathscr{L}^{2}$ with compact support. Since the aggregate of those functions is dense in $\mathscr{L}_{t}^{2},(3.15)$ is valid for every $f \in \mathscr{L}_{t}^{2}$ in view of Proposition 3.1, (1). Finally we shall prove

$$
\int_{\mathbb{R}^{3}}|Q(x) R(\bar{z}) f| d x<+\infty
$$

for $f \in \mathscr{L}^{2}$ with compact support. Put $u=R(\bar{z}) f$. Then we have

$$
<\left(L_{0}+Q(x)\right) u(x), u(x)>-\bar{z}|u(x)|^{2}=<f(x), u(x)>\text {. }
$$


Integrating (3.17) by parts over $r_{0} \leqq|x| \leqq R\left(r_{0}\right.$ is sufficiently large so that $S_{r_{0}}=\left\{x ;|x|=r_{0}\right\}$ encloses the support of $\left.f(x)\right)$ and taking the imaginary part, we obtain

$$
\begin{gathered}
(\operatorname{Im} z) \int_{r_{0} \leqq|x| \leqq R}|u(x)|^{2} d x \\
=\frac{R^{2}}{2} \int_{S}<\sum_{j=1}^{3} \omega_{j} \alpha_{j} u(R \omega), u(R \omega)>d \omega \\
-\frac{r_{0}^{2}}{2} \int_{S}<\sum_{j=1}^{3} \omega_{j} \alpha_{j} u\left(r_{0} \omega\right), u\left(r_{0} \omega\right)>d \omega .
\end{gathered}
$$

Since $u \in \mathscr{L}^{2}$ and, consequently, there exists a sequence $\left\{R_{j}\right\}$ such that $R_{j} \rightarrow \infty$ and $R_{j}^{2} \int_{S}\left|u\left(R_{j} \omega\right)\right|^{2} d \omega \rightarrow 0(j \rightarrow \infty)$, we have from (3.18) that

$$
\begin{aligned}
& (\operatorname{Im} z) \int_{|x| \geqq r_{0}}|u(x)|^{2} d x \\
& \quad=-\frac{r_{0}^{2}}{2} \int_{S}<\sum_{j=1}^{3} \omega_{j} \alpha_{j} u\left(r_{0} \omega\right), u\left(r_{0} \omega\right)>d \omega .
\end{aligned}
$$

This implies that

$$
\int_{|x| \geqq r_{0}}|u(x)|^{2} d x \leqq \frac{\text { const. }}{|\operatorname{Im} z|} r_{0}^{2} \int_{S}\left|u\left(r_{0} \omega\right)\right|^{2} d \omega
$$

and, by integrating (3.20) with respect to $r_{0}$ from $r$ to infinity,

$$
\int_{|x| \geqq r}(|x|-r)|u(x)|^{2} d x \leqq \frac{\text { const. }}{|\operatorname{Im} z|} \int_{|x| \geqq r}|u(x)|^{2} d x .
$$

Repeating the integration we see

$$
\int_{\mathbf{R}^{3}}|x|^{m}|u(x)|^{2} d x<+\infty \quad(m=1,2, \ldots)
$$

Since $Q(x)$ is bounded in $|x| \geqq R_{0}$ (see the assumption (A) in $\S 2$ ), we have

$$
\begin{aligned}
& \int_{|x| \geqq R_{0}}|Q(x) u(x)| d x \\
\leqq & \text { const. }\left(\int_{|x| \geqq R_{0}}(1+|x|)^{-m} d x\right)^{1 / 2}\left(\int_{|x| \geqq R_{0}}(1+|x|)^{m}|u(x)|^{2} d x\right)^{1 / 2},
\end{aligned}
$$


where $m>3$. Proposition 2.3 yields that

$$
Q(x) u(x)=Q(x) R(\bar{z}) f \in \mathscr{L}_{t}^{2}
$$

and, accordingly, that $Q(x) u(x)$ is locally integrable. Hence we obtain

$$
\int_{|x| \leqq R_{0}}|Q(x) u(x)| d x<+\infty
$$

Thus (3.16) follows from these estimates.

Q.E.D.

The following fact is obtained directly from Proposition 3.1, (1), and Lemma 3.7.

Lemma 3.8. For each $J_{+}(a, b)\left(J_{-}(a, b)\right), 0<\theta<t-1 / 2$ and $0<\alpha<\beta$ there exist positive constants $C_{1}=C_{1}(t), C_{2}=C_{2}(t, \theta)$ and $C_{3}=C_{3}(t)$ such that

$$
\begin{gathered}
\left\|A_{v}^{*}(z, r) f\right\|_{\mathscr{L}^{2}(S)} \leqq C_{1}\|f\|_{\mathscr{L}_{t}^{2}}, \\
\left\|A_{v}^{*}\left(z, r_{1}\right) f-A_{v}^{*}\left(z, r_{2}\right) f\right\|_{\mathscr{L}^{2}(S)} \leqq C_{2}\left|r_{1}-r_{2}\right|^{\theta}\|f\|_{\mathscr{L}_{t}^{2}}
\end{gathered}
$$

and

$$
\left\|A_{v}^{*}\left(z_{1}, r\right) f-A_{v}^{*}\left(z_{2}, r\right) f\right\|_{\mathscr{L}^{2}(S)} \leqq C_{3} \|\left(R\left(\overline{\left.z_{1}\right)}-R\left(\overline{z_{2}}\right)\right) f \|_{\mathscr{P}_{-t}^{1}}\right.
$$

for $f \in \mathscr{L}_{t}^{2}, \alpha \leqq r, r_{1}, r_{2} \leqq \beta, z, z_{1}, z_{2} \in J_{+}(a, b)\left(J_{-}(a, b)\right)$.

Corollary 3.9. For $f \in \mathscr{L}_{t}^{2}$ and $r>0$

$$
\left\|A_{v}^{*}(z, r) f-A_{v}^{*}(\lambda \pm 0 i, r) f\right\|_{\mathscr{L}^{2}(S)} \longrightarrow 0 \quad(z \rightarrow \lambda \pm 0 i)
$$

and

$$
A_{v}^{*}(\lambda \pm 0 i, r) f=(2 \pi)^{3 / 2}\left\{\left(Z_{v}^{0} f\right)(r \cdot)-\left(Z_{v}^{0} Q R^{\mp}(\lambda) f\right)(r \cdot)\right\} \text {. }
$$

(3.22)-(3.24) of Lemma 3.8 hold with $z, z_{1}, z_{2} \in \overline{J_{+}(a, b)} \overline{\left(J_{-}(a, b)\right)}$.

Proof. As a result of Corollary 3.4, $A_{v}(z, r) h$ converges in $\mathscr{H}_{-}^{1}$ t to $A_{v}(\lambda \pm 0 i, r) h$ as $z \rightarrow \lambda \pm 0 i$ for each $h \in \mathscr{L}^{2}(S)$, which suggests that $A_{v}^{*}(z, r) f$ tends weakly in $\mathscr{L}^{2}(S)$ to $A^{*}(\lambda \pm 0 i, r) f$ for $f \in \mathscr{L}_{t}^{2}$. On the 
other hand $A_{v}^{*}(z, r) f$ is a Cauchy sequence in $\mathscr{L}^{2}(S)$ for $f \in \mathscr{L}_{t}^{2}$ by means of Proposition 2.2 and (3.24) in Lemma 3.8. Thus we have

$$
\left\|A_{v}^{*}(z, r) f-A_{v}^{*}(\lambda \pm 0 i, r) f\right\|_{\mathscr{L}^{2}(S)} \longrightarrow 0 \quad(z \rightarrow \lambda \pm 0 i)
$$

for $f \in \mathscr{L}_{t}^{2}$. The last assertion of our corollary follows from the limiting procedure.

Lemma 3.10. Let $\operatorname{Im} z \neq 0$. Then

$$
\widehat{R(z) f}(r \cdot)=(2 \pi)^{-3 / 2} \sum_{v=p, n} \frac{1}{\tau_{v} \sqrt{r^{2}+1}-z} A_{v}^{*}(\bar{z}, r) f
$$

holds for every $f \in \mathscr{L}_{t}^{2}$.

Proof. Lemma 3.5 and Proposition 3.6, (2), lead us to

$$
\begin{aligned}
& (R(\bar{z}) g, f)_{\mathscr{L}^{2}}=\left(V_{p}(\bar{z}) g+V_{n}(\bar{z}) g, f\right)_{\mathscr{L}^{2}} \\
= & \left((2 \pi)^{-3 / 2} \int_{0}^{+\infty}\left(\frac{A_{p}(\bar{z}, r)}{\sqrt{r^{2}+1}-\bar{z}}-\frac{A_{n}(\bar{z}, r)}{\sqrt{r^{2}+1}+\bar{z}}\right) \hat{g}(r \cdot) r^{2} d r, f\right)_{\mathscr{L}^{2}} \\
= & (2 \pi)^{-3 / 2} \int_{0}^{+\infty}\left(\left(\frac{A_{p}(\bar{z}, r)}{\sqrt{r^{2}+1}-\bar{z}}-\frac{A_{n}(\bar{z}, r)}{\sqrt{r^{2}+1}+\bar{z}}\right) \hat{g}(r \cdot), f\right)_{\mathscr{L}^{2}} r^{2} d r \\
= & (2 \pi)^{-3 / 2} \int_{0}^{+\infty}\left(\hat{g}(r \cdot),\left(\frac{A_{p}^{*}(\bar{z}, r)}{\sqrt{r^{2}+1}-z}-\frac{A_{n}^{*}(\bar{z}, r)}{\sqrt{r^{2}+1}+z}\right) f\right)_{\mathscr{L}^{2}(S)} r^{2} d r
\end{aligned}
$$

for $\hat{g} \in \mathscr{C}_{0}^{\infty}\left(\mathbb{R}^{3}-\{0\}\right)$. Noting that

$$
(R(\bar{z}) f, g)_{\mathscr{S}^{2}}=(g, R(z) f)_{\mathscr{S}^{2}}=(\hat{g}, \widehat{R(z) f})_{\mathscr{S}^{2}}
$$

we can complete the proof in view of the arbitrariness of $\hat{g}$ Q.E.D.

\section{§4. Expansion Theorems}

Let $E(\lambda)\left(E_{0}(\lambda)\right)$ be the right-continuous resolution of the identity associated with the selfadjoint operator $H=H_{0}+Q\left(H_{0}\right)$. We know that $H$ has at most a countable number of discrete eigenvalues in $(-1,+1)$ (Proposition 2.1). We denote by $\mu_{1}, \mu_{2}, \ldots$ the eigenvalues of $H$ in $[-1$, $+1]$, counted repeatedly according to the multiplicity, and by $\varphi_{1}, \varphi_{2}, \ldots$ 
orthonormalized eigenfunctions for $\mu_{1}, \mu_{2}, \ldots$

Definition 4.1. Let $F_{v}^{ \pm}(r)=A_{v}\left(\tau_{v} \sqrt{r^{2}+1} \mp 0 i, r\right)$ for $r>0$. We define the generalized Fourier transform $\hat{f}_{v}^{ \pm}(r)$ of $f \in \mathscr{L}_{t}^{2}$ by

$$
\hat{f}_{v}^{ \pm}(r)=(2 \pi)^{-3 / 2} F_{v}^{ \pm}(r)^{*} f
$$

Theorem 4.2. For $f \in \mathscr{L}_{t}^{2}$

$$
\begin{aligned}
\|f\|_{\mathscr{L}^{2}}^{2}=\int_{0}^{+\infty}\left\|\hat{f}_{p}^{ \pm}(r)\right\|_{\mathscr{L}^{2}(S)}^{2} r^{2} d r+\int_{0}^{+\infty}\left\|\hat{f}_{n}^{ \pm}(r)\right\|_{\mathscr{L}^{2}(S)}^{2} r^{2} d r \\
+\sum_{j}\left|\left(f, \varphi_{j}\right)\right|^{2}
\end{aligned}
$$

Proof. We shall show for $1<\alpha<\beta<+\infty$ and $-\infty<\alpha^{\prime}<\beta^{\prime}<-1$ that

$$
\begin{aligned}
& ((E(\beta)-E(\alpha)) f, f)=\int_{\alpha \leqq \sqrt{r^{2}+1} \leqq \beta}\left\|\hat{f}_{p}^{+}(r)\right\|_{\mathscr{L}^{2}(S)}^{2} r^{2} d r \\
& \left(\left(E\left(\beta^{\prime}\right)-E\left(\alpha^{\prime}\right)\right) f, f\right)=\int_{-\beta^{\prime} \leqq \sqrt{r^{2}+1} \leqq-\alpha^{\prime}}\left\|\hat{f}_{n}^{+}(r)\right\|_{\mathscr{L}^{2}(S)}^{2} r^{2} d r
\end{aligned}
$$

and

$$
((E(1)-E(-1-0)) f, f)=\sum_{j}\left|\left(f, \varphi_{j}\right)\right|^{2},
$$

from which our assertion (with the upper sign) follows, if we bring $\alpha, \alpha^{\prime}, \beta, \beta^{\prime}$ to $+1,-\infty,+\infty,-1$, respectively ((4.1) with the lower sign is similarly obtained). To prove (4.2) we make use of

$$
\begin{gathered}
1 / 2((E(\beta)+E(\beta-0)) f, f)-1 / 2((E(\alpha)+E(\alpha-0)) f, f) \\
=\frac{1}{2 \pi i} \lim _{\eta \downarrow 0} \int_{\alpha}^{\beta}(R(\mu+i \eta) f-R(\mu-i \eta) f, f) d \mu .
\end{gathered}
$$

Since $(E(\lambda) f, f)$ is (absolutely) continuous with respect to $\lambda$ in $(-\infty$, -1 ) and $(+1,+\infty)$ (see Proposition 2.1), we have, using the resolvent equation $R\left(z_{1}\right)-R\left(z_{2}\right)=\left(z_{1}-z_{2}\right) R\left(z_{1}\right) R\left(z_{2}\right)$, that

$$
\begin{aligned}
& ((E(\beta)-E(\alpha)) f, f)=\frac{1}{2 \pi i} \lim _{\eta \downarrow 0} \int_{\alpha}^{\beta} 2 \eta i(R(\mu-i \eta) R(\mu+i \eta) f, f) d \mu \\
& \quad=\frac{1}{\pi} \lim _{\eta \downarrow 0} \int_{\alpha}^{\beta} \eta\|R(\mu+i \eta) f\|^{2} d \mu=\frac{1}{\pi} \lim _{\eta \downarrow 0} \int_{\alpha}^{\beta} \eta\|\widehat{R(\mu+i \eta) f}\|^{2} d \mu
\end{aligned}
$$


Now let us select a non-negative $C_{0}^{\infty}$-function $\gamma(x)=\gamma(|x|)$ such that

$$
\gamma(x) \begin{cases}=1, & \sqrt{\alpha^{2}-1}-\delta \leqq|x| \leqq \sqrt{\beta^{2}-1}+\delta, \\ =0, & |x| \leqq \sqrt{\alpha^{2}-1}-2 \delta, \\ \leqq 1, & \text { otherwise }\end{cases}
$$

where $\delta$ is a sufficiently small, fixed, positive number. Then we have

$$
\begin{aligned}
\text { (4.5) } & =\frac{1}{\pi} \lim _{\eta \downarrow 0}\left\{\eta \int_{\alpha}^{\beta}\|\sqrt{\gamma R(\mu+i \eta) f}\|^{2} d \mu+\eta \int_{\alpha}^{\beta}\|\sqrt{1-\gamma} \widehat{R(\mu+i \eta) f}\|^{2} d \mu\right\} \\
& \equiv \frac{1}{\pi} \lim _{\eta \downarrow 0}\left(I_{1}(\eta)+I_{2}(\eta)\right) .
\end{aligned}
$$

We shall derive

$$
\left\{\begin{array}{l}
\lim _{\eta \downarrow 0} I_{1}(\eta)=\pi \int_{\alpha \leqq \sqrt{r^{2}+1} \leqq \beta}\left\|\hat{f}_{p}^{+}(r)\right\|_{\mathscr{L}^{2}(S)}^{2} r^{2} d r \\
\lim _{\eta \downarrow 0} I_{2}(\eta)=0
\end{array}\right.
$$

First we prove that $\lim _{\eta \downarrow 0} I_{2}(\eta)=0$.

Set $u=u(\mu, \eta, f)=R(\mu+i \eta) f$, so that

$$
\left(H_{0}-\mu-i \eta\right) u=f-Q u .
$$

On the other hand it follows from Propositions 2.2 and 2.3 that

$$
\|Q u(\mu, \eta, f)\|_{\mathscr{L}_{t}^{2}} \leqq C_{1}\|f\|_{\mathscr{L}_{t}^{2}},
$$

where the constant $C_{1}$ does not depend on $\mu+i \eta \in J_{+}(\alpha, \beta)$. Therefore, taking the Fourier transforms of both sides of (4.7) leads us to

$$
\begin{aligned}
& \|\sqrt{1-\gamma} \widehat{R(\mu+i \eta) f}\|=\|\sqrt{1-\gamma \hat{u}}\| \\
& \quad=\left\|\sqrt{1-\gamma} \frac{\hat{L}_{0}(\xi)+\mu+i \eta}{|\xi|^{2}+1-(\mu+i \eta)^{2}}\left(\hat{f}(\xi)-(Q u)^{\wedge}(\xi)\right)\right\| \\
& \leqq C_{2}\|f\|_{\mathscr{L}_{t}^{2}},
\end{aligned}
$$

where $C_{2}=C_{2}(\alpha, \beta)$ is independent of $\mu+i \eta \in J_{+}(\alpha, \beta)$ as a result of our 
choice of $\gamma(x)$. Hence we obtain

$$
I_{2}(\eta)=\eta \int_{\alpha}^{\beta} \sqrt{1-\gamma R(\mu+i \eta) f}\left\|^{2} d \mu \leqq \eta C_{2}(\beta-\alpha)\right\| f \|_{\mathscr{L}_{t}^{2}}
$$

which shows $\lim _{\eta \downarrow 0} I_{2}(\eta)=0$.

To study $I_{1}(\eta)$ we prepare a proposition (see Ikebe [7], p. 25, where the same result appears).

Proposition 4.3. Let $f(\mu, \eta)$ be a continuous function defined on $[\alpha, \beta] \times\left[0, \eta_{0}\right]\left(\alpha<\beta, \eta_{0}>0\right)$. Then the following relation holds;

$$
\frac{1}{\pi} \lim _{\eta \downarrow 0} \int_{\alpha}^{\beta} \frac{\eta}{(\lambda-\mu)^{2}+\eta^{2}} f(\mu, \eta) d \mu=\left\{\begin{array}{c}
0, \quad \lambda>\beta \text { or } \lambda<\alpha \\
\frac{f(\lambda, 0)}{2}, \lambda=\alpha \text { or } \beta \\
f(\lambda, 0), \alpha<\lambda<\beta .
\end{array}\right.
$$

Let us return to the proof of (4.6). From Lemma 3.10 we get

$$
\begin{gathered}
\|\sqrt{\gamma} \widehat{R(\mu+i \eta) f}\|^{2} \\
=(2 \pi)^{-3} \int_{0}^{+\infty} \| \sum_{v=p, n} \frac{\sqrt{\gamma}}{\tau_{v} \sqrt{r^{2}+1}-\mu-i \eta} A_{v}^{*}(\mu-i \eta, r) f{\| \mathscr{L}^{2}(S)}_{r^{2}} d r \\
=(2 \pi)^{-3} \int_{0}^{+\infty}\left\{\frac{\gamma(r)}{\left(\sqrt{r^{2}+1}-\mu\right)^{2}+\eta^{2}}\left\|A_{p}^{*}(\mu-i \eta, r) f\right\|_{\mathscr{L}^{2}(S)}^{2}\right. \\
\left.\quad+\frac{\gamma(r)}{\left(\sqrt{r^{2}+1}+\mu\right)^{2}+\eta^{2}}\left\|A_{n}^{*}(\mu-i \eta, r) f\right\|_{\mathscr{L}^{2}(S)}^{2}\right\} r^{2} d r,
\end{gathered}
$$

where we have used

$$
\left(A_{p}^{*}(z, r) f, A_{n}^{*}(z, r) f\right)_{\mathscr{L}^{2}(S)}=\left(A_{n}^{*}(z, r) f, A_{p}^{*}(z, r) f\right)_{\mathscr{L}^{2}(S)}=0,
$$

which follows from (1.2) in $\S 1$ and (3.15) in Lemma 3.7. In view of Proposition 4.3 and Lemma 3.8 we have

$$
\int_{\alpha}^{\beta} \frac{\eta}{\left(\sqrt{r^{2}+1}+\mu\right)^{2}+\eta^{2}}\left\|A_{n}^{*}(\mu-i \eta, r) f\right\|_{\mathscr{L}^{2}(S)}^{2} d \mu \longrightarrow 0
$$


and that

$$
\int_{\alpha}^{\beta} \frac{\eta}{\left(\sqrt{r^{2}+1}-\mu\right)^{2}+\eta^{2}}\left\|A_{p}^{*}(\mu-i \eta, r) f\right\|_{\mathscr{L}^{2}(S)}^{2} d \mu
$$

is uniformly bounded for $0<\eta \leqq 1$ and $r \in \operatorname{supp} \gamma$, and converges to

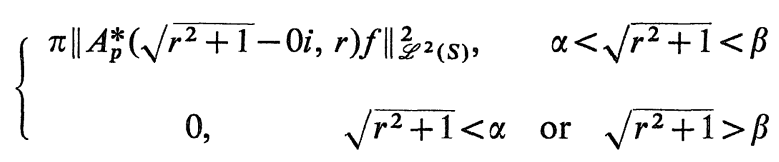

as $\eta \downarrow 0$. Therefore we obtain from Fubini's theorem and Lebesgue's convergence theorem that

$$
\begin{aligned}
& \lim _{\eta \downarrow 0} I_{1}(\eta) \\
& =(2 \pi)^{-3} \int_{0}^{+\infty} \gamma(r) r^{2} d r \lim _{\eta \downarrow 0} \int_{\alpha}^{\beta}\left\{\frac{\eta}{\left(\sqrt{r^{2}+1}-\mu\right)^{2}+\eta^{2}}\left\|A_{p}^{*}(\mu-i \eta, r) f\right\|_{\mathscr{L}^{2}(S)}^{2}\right. \\
& \left.+\frac{\eta}{\left(\sqrt{r^{2}+1}+\mu\right)^{2}+\eta^{2}}\left\|A_{n}^{*}(\mu-i \eta, r) f\right\|_{\mathscr{L}^{2}(S)}^{2}\right\} d \mu \\
& =\pi \int_{\alpha<\sqrt{r^{2}+1}<\beta}\left\|\hat{f}_{p}^{+}(r)\right\|_{\mathscr{L}^{2}(S)}^{2} r^{2} d r .
\end{aligned}
$$

The argument for (4.3) is almost the same as the above. (4.4) reduces to the well-known Parseval's equality of Fourier series.

Q.E.D.

The above result shows us that

$$
\left\|\hat{f}_{v}^{ \pm}\right\|_{\mathscr{L}^{2}}=\int_{0}^{+\infty}\left\|\hat{f}_{v}^{ \pm}(r)\right\|_{\mathscr{L}^{2}(S)}^{2} r^{2} d r \leqq\|f\|_{\mathscr{L}^{2}}^{2} \quad(v=p, n)
$$

for $f \in \mathscr{L}_{t}^{2}$. Since $\mathscr{L}_{t}^{2}$ is dense in $\mathscr{L}^{2}$, there exists a bounded operator $Z_{v}^{ \pm}$in $\mathscr{L}^{2}$ such that

$$
\left(Z_{v}^{ \pm} f\right)(r \omega)=\left(\hat{f}_{v}^{ \pm}(r)\right)(\omega)=(2 \pi)^{-3 / 2}\left(F_{v}^{ \pm}(r)^{*} f\right)(\omega) \quad(\omega \in S)
$$

for $f \in \mathscr{L}_{t}^{2}$.

Theorem 4.4. $Z_{v}^{ \pm}$is a partially isometric operator on $\mathscr{L}^{2}$ with the range in $\psi_{v} \mathscr{L}^{2}$ such that 


$$
\left\|Z_{p}^{ \pm} f\right\|=\|(I-E(1)) f\|, \quad\left\|Z_{n}^{ \pm} f\right\|=\|E(-1-0) f\|
$$

for $f \in \mathscr{L}^{2}$, or equivalently

$$
\left(Z_{p}^{ \pm}\right) * Z_{p}^{ \pm}=I-E(1), \quad\left(Z_{n}^{ \pm}\right) * Z_{n}^{ \pm}=E(-1-0) .
$$

For $f \in \mathscr{L}^{2}$

$$
\begin{aligned}
& \|f\|^{2}=\left\|Z_{p}^{ \pm} f\right\|^{2}+\left\|Z_{n}^{ \pm} f\right\|^{2}+\sum_{j}\left|\left(f, \varphi_{j}\right)\right|^{2}, \\
& f=\left(Z_{p}^{ \pm}\right)^{*} Z_{p}^{ \pm} f+\left(Z_{n}^{ \pm}\right)^{*} Z_{n}^{ \pm} f+\sum_{j}\left(f, \varphi_{j}\right) \varphi_{j}
\end{aligned}
$$

Let $g$ be any $\mathscr{L}^{2}$-function with compact support not containing the origin. Then the $\mathscr{H}_{-}^{1}{ }_{t}$-valued Bochner integral $\int_{0}^{+\infty} F_{v}^{ \pm}(r) g(r \cdot) r^{2} d r$ exists and belongs to $\mathscr{L}^{2}$, satisfying

$$
\left(Z_{v}^{ \pm}\right)^{*} g=(2 \pi)^{-3 / 2} \int_{0}^{+\infty} F_{v}^{ \pm}(r) g\left(r^{\circ}\right) r^{2} d r
$$

Proof. Since $\mathscr{L}_{t}^{2}$ is dense in $\mathscr{L}^{2}$, we have from (4.2), (4.3) and (4.4) that

$$
\|(I-E(1)) f\|=\left\|Z_{p}^{ \pm} f\right\|, \quad\|E(-1-0) f\|=\left\|Z_{n}^{ \pm} f\right\|
$$

and

$$
\|(E(1)-E(-1-0)) f\|^{2}=\sum_{j}\left|\left(f, \varphi_{j}\right)\right|^{2}
$$

for $f \in \mathscr{L}^{2}$. (4.10)-(4.13) follow directly from these relations. As $\left(\hat{f}_{v}^{ \pm}(r)\right)(\omega)\left(f \in \mathscr{L}_{t}^{2}\right)$ is in $\psi_{v} \mathscr{L}^{2}$ by $\mathbb{L e m m a} 3.7$, the range of $Z_{v}^{ \pm}$is included in $\psi_{v} \mathscr{L}^{2}$, for the range of a partially isometric operator is closed (see Kato [9], p. 258). In order to prove our last statement (4.14) we have first to see that the integral exists. For the strong measurability we need only to assure the weak one by means of Proposition 3.6, (1), for $\mathscr{H}_{-t}^{1}$ is separable. For each $f \in \mathscr{L}_{t}^{2}$

$$
\left(F_{v}^{ \pm}(r) g(r \cdot), f\right)_{\mathscr{L}^{2}}=\left(g(r \cdot), F_{v}^{ \pm}(r)^{*} f\right)_{\mathscr{L}^{2}(S)} \quad(r>0)
$$

is a Lebesgue measurable function with respect to $r$ by virtue of Corollary 3.9. For $f \in \mathscr{H}_{t}^{-1}$ there exists a sequence $\left\{f_{j}\right\} \subset \mathscr{L}_{t}^{2}$ such that $\left\|f-f_{j}\right\|_{\mathscr{C}_{t}^{-1}} \rightarrow 0(j \rightarrow \infty)$. Then 


$$
\left(g(r \cdot), F_{v}^{ \pm}(r)^{*} f\right)_{\mathscr{L}^{2}(S)}=\lim _{j \rightarrow \infty}\left(g(r \cdot), F_{v}^{ \pm}(r)^{*} f_{j}\right)_{\mathscr{L}^{2}(S)}
$$

is measurable as a limit of measurable functions convergent almost everywhere on $(0,+\infty)$. Thus we have proved the measurability. Since the compact support of $g$ has no intersection with the origin, we obtain

$$
\begin{aligned}
\int_{0}^{+\infty}\left\|F_{v}^{ \pm}(r) g(r \cdot)\right\|_{\mathscr{H}^{1}-t} r^{2} d r & \leqq \text { const. } \int_{0}^{+\infty}\|g(r \cdot)\|_{\mathscr{L}^{2}(S)} r^{2} d r \\
& \leqq \text { const. }\|g\|_{\mathscr{L}^{2}},
\end{aligned}
$$

where the constants depends on the support of $g$. This guarantees the existence of the Bochner integral $\int_{0}^{+\infty} F_{v}^{ \pm}(r) g(r \cdot) r^{2} d r$. Then (4.14) is seen from

$$
\begin{aligned}
\left(Z_{v}^{ \pm} f, g\right) & =\left(\hat{f}_{v}^{ \pm}, g\right) \\
& =(2 \pi)^{-3 / 2} \int_{0}^{+\infty}\left(F_{v}^{ \pm}(r)^{*} f, g(r \cdot)\right)_{\mathscr{L}^{2}(S)} r^{2} d r \\
& =\left(f,(2 \pi)^{-3 / 2} \int_{0}^{+\infty} F_{v}^{ \pm}(r) g(r \cdot) r^{2} d r\right)_{\mathscr{S}^{2}}
\end{aligned}
$$

for $f \in \mathscr{L}_{i}^{2}$.

Q.E.D.

$Z_{p}^{ \pm}$and $Z_{n}^{ \pm}$have the following integral representation with generalized eigenfunctions.

Theorem 4.5. Let $\Sigma=\left\{y_{j}\right\}_{j=1,2, \ldots, \sigma}$ be the set of all the singurarities of $Q(x)$. Then there exists a $4 \times 4$ matrix $\Phi_{v}^{ \pm}(x, r, \omega)$ such that

$$
F_{v}^{ \pm}(r) h=\int_{S} \Phi_{v}^{ \pm}(x, r, \omega) h(\omega) d \omega
$$

for $h \in \mathscr{L}^{2}(S) . \quad \Phi_{v}^{ \pm}(x, r, \cdot)$ consists of $L^{2}(S)$-components, locally Hölder continuous in $L^{2}(S)$ with respect to $x \in \mathbb{R}^{3}-\Sigma$ and uniformly bounded in $L^{2}(S)$ for $\alpha \leqq r \leqq \beta(0<\alpha<\beta<\infty)$. $Z_{v}^{ \pm}$is written as

$$
\left(Z_{v}^{ \pm} f\right)(r \cdot)=(2 \pi)^{-3 / 2} \int_{\mathbf{R}^{3}} \Phi_{v}^{ \pm}(x, r, \cdot)^{*} f(x) d x
$$

for $f \in \mathscr{L}^{2}$ with compact support in $\mathbf{R}^{3}-\Sigma . \Phi_{v}^{ \pm}(x, r, \omega)$ may be called generalized eigenfunctions in the following sense: 


$$
\begin{aligned}
\left(L_{0}\right. & +Q(x)) \int_{S} \Phi_{v}^{ \pm}(x, r, \omega) h(\omega) d \omega \\
& =\tau_{v} \sqrt{r^{2}+1} \int_{S} \Phi^{ \pm}(x, r, \omega) h(\omega) d \omega
\end{aligned}
$$

for any $h \in \mathscr{L}^{2}(S)$.

Proof. Let us take an arbitrary $\gamma(x) \in C_{0}^{\infty}\left(\mathbb{R}^{3}-\Sigma\right)$ such that $\gamma(x)=1$, $x \in J_{a}$, for a positive number $a$, where

$$
J_{a}=\left\{x \in \mathbb{R}^{3} ;|x| \leqq a \text { and } \max _{1 \leqq j \leqq \sigma}\left|x-y_{j}\right| \geqq \frac{1}{a}\right\} .
$$

We recall the definition

$$
\begin{aligned}
F_{v}^{ \pm}(r) h & =A\left(\tau_{v} \sqrt{r^{2}+1} \mp 0 i\right) h \\
& =\left(I-R^{\mp}\left(\tau_{v} \sqrt{r^{2}+1}\right) Q\right) \int_{S} \Phi_{v}^{0}(x, r \omega) h(\omega) d \omega
\end{aligned}
$$

for $h \in \mathscr{L}^{2}(S)$. Multiplying the above equation by $\gamma(x)$ and operating $L_{0}$, we have

$$
\begin{aligned}
& L_{0}\left(\gamma F_{v}^{ \pm}(r) h\right) \\
& \quad=\gamma\left\{\tau_{v} \sqrt{r^{2}+1}-Q(x)+\left(Q(x)-\tau_{v} \sqrt{r^{2}+1}\right) R^{\mp}\left(\tau_{v} \sqrt{r^{2}+1}\right) Q\right\} \\
& \int_{S} \Phi_{v}^{0}(x, r \omega) h(\omega) d \omega+\frac{1}{i} \sum_{j=1}^{3} \alpha_{j} \frac{\partial \gamma}{\partial x_{j}} F_{v}^{ \pm}(r) h
\end{aligned}
$$

and, consequently,

$$
\left\|L_{0}\left(\gamma F_{v}^{ \pm}(r) h\right)\right\|_{\mathscr{L}^{2}} \leqq C_{1}\|h\|_{\mathscr{L}^{2}(S)}
$$

for $\alpha \leqq r \leqq \beta$, where $C_{1}$ is independent of $h \in \mathscr{L}^{2}(S)$ and $\alpha \leqq r \leqq \beta$. We operate $L_{0}$ again to (4.18) and use the differentiability of $Q(x)$ except on $\Sigma$. Then we have in the same manner

$$
\left\|L_{0}^{2}\left(\gamma F_{v}^{ \pm}(r) h\right)\right\|_{\mathscr{L}^{2}} \leqq C_{2}\|h\|_{\mathscr{L}^{2}(S)} .
$$

Sobolev's imbedding theorem (Proposition 3.1, (2)) and $\mathbb{L}_{0}^{2}=-\Delta+1$ imply that 


$$
\left|\left(F_{v}^{ \pm}(r) h\right)(x)\right| \leqq C_{3}\left\|\gamma F_{v}^{ \pm}(r) h\right\|_{\mathscr{C}^{2}} \leqq C_{4}\|h\|_{\mathscr{L}^{2}(S)}
$$

and

$$
\left|\left(F_{v}^{ \pm}(r) h\right)(x)-\left(F_{v}^{ \pm}(r) h\right)\left(x^{\prime}\right)\right| \leqq C_{5}(\theta)\left|x-x^{\prime}\right|^{\theta}\|h\|_{\mathscr{Z}^{2}(S)}
$$

for $x, x^{\prime} \in J_{a}, \alpha \leqq r \leqq \beta$ and $0<\theta<1 / 2$. It follows from (4.20) that the mapping

$$
h \longrightarrow\left(F_{v}^{ \pm}(r) h\right)_{m}(x) \quad(m=1,2,3,4)
$$

is a bounded linear functional on $\mathscr{L}^{2}(S)$ for each $x \in \mathbb{R}^{3}-\Sigma$ and $r>0$, $z_{m}$ denotes the $m$-th component of $z$. According to Riesz's representation theorem there exists a vector $\varphi_{v}^{ \pm}, m(x, r, \cdot) \in \mathscr{L}^{2}(S)\left(x \in \mathbb{R}^{3}-\Sigma, r>0\right)$ such that

$$
\left(F_{v}^{ \pm}(r) h\right)_{m}(x)=\int_{S}<h(\omega), \varphi_{v, m}^{ \pm}(x, r, \omega)>d \omega .
$$

So we set

$$
\Phi_{v}^{ \pm}(x, r, \omega)=\left(\begin{array}{l}
\overline{\varphi_{v, 1}^{ \pm}(x, r, \omega)} \\
\overline{\varphi_{v, 2}^{ \pm}(x, r, \omega)} \\
\overline{\varphi_{v, 3}^{ \pm}(x, r, \omega)} \\
\overline{\varphi_{v, 4}^{ \pm}(x, r, \omega)}
\end{array}\right)
$$

which yields (4.15). (4.20) and (4.22) give us that

$$
\begin{aligned}
& \int_{S}\left|\Phi_{v}^{ \pm}(x, r, \omega)\right|^{2} d \omega=\sum_{m=1}^{4} \int_{S}\left|\varphi_{v, m}^{ \pm}(x, r, \omega)\right|^{2} d \omega \\
& =\sum_{m=1}^{4}\left(F_{v}^{ \pm}(r) \varphi_{v}^{ \pm},\right)_{m}(x) \leqq C_{6}\left(\int_{S}\left|\Phi_{v}^{ \pm}(x, r, \omega)\right|^{2} d \omega\right)^{1 / 2} \\
& \quad\left(x \in J_{a}, \alpha \leqq r \leqq \beta\right) .
\end{aligned}
$$

Therefore we have

$$
\left\|\Phi_{v}^{ \pm}(x, r, \cdot)\right\|_{\mathscr{L}^{2}(S)} \leqq C_{6}
$$

for $x \in J_{a}$ and $\alpha \leqq r \leqq \beta$. Similarly we get from (4.21) 


$$
\left\|\Phi_{v}^{ \pm}(x, r, \cdot)-\Phi_{v}^{ \pm}\left(x^{\prime}, r, \cdot\right)\right\|_{\mathscr{L}^{2}(S)} \leqq C_{7}(\theta)\left|x-x^{\prime}\right|^{e}
$$

for $x, x^{\prime} \in J_{a}$ and $\alpha \leqq r \leqq \beta$ (the idea of the above proof is due to Agmon [2], Lemma 2.1). In order to see (4.16) we take any $f \in \mathscr{L}^{2}$ with compact support in some $J_{a}$. Then we have

$$
\begin{aligned}
\left(F_{v}^{ \pm}(r) h, f\right)_{\mathscr{L}^{2}}=\left(\int_{S} \Phi_{v}^{ \pm}(\cdot, r, \omega) h(\omega) d \omega, f\right)_{\mathscr{L}^{2}} \\
\quad=\left(h, \int_{\mathbf{R}^{3}} \Phi_{v}^{ \pm}(x, r, \cdot)^{*} f(x) d x\right)_{\mathscr{L}^{2}(S)}
\end{aligned}
$$

where we have used Fubini's theorem, which is possible because

$$
\begin{aligned}
\int_{\mathbb{R}^{3}} \int_{S}\left|\Phi_{v}^{ \pm}(x, r, \omega)\right||h(\omega)||f(x)| d \omega d x & \\
& \leqq\|h\|_{\mathscr{L}^{2}(S)} \sup _{x \in \operatorname{supp}[f]}\left\|\Phi_{v}^{ \pm}\left(x, r^{\cdot}\right)\right\|_{\mathscr{L}^{2}(S)} \int_{\mathbb{R}^{3}}|f(x)| d x<+\infty
\end{aligned}
$$

Thus we obtain

$$
\left(Z_{v}^{ \pm} f\right)(r \cdot)=(2 \pi)^{-3 / 2} F_{v}^{ \pm}(r)^{*} f=(2 \pi)^{-3 / 2} \int_{\mathbb{R}^{3}} \Phi_{v}^{ \pm}(x, r, \cdot)^{*} f(x) d x
$$

(4.17) is seen by substituting $\tau_{v} \sqrt{r^{2}+1} \mp 0 i$ for $z$ in (3.4):

$$
\begin{aligned}
& \left(L_{0}+Q(x)\right) \int_{S} \Phi_{v}^{ \pm}(x, r, \omega) h(\omega) d \omega \\
& \quad=\left(L_{0}+Q(x)\right) F_{v}^{ \pm}(r) h=\left(L_{0}+Q(x)\right) A_{v}\left(\tau_{v} \sqrt{r^{2}+1} \mp 0 i, r\right) h \\
& =\tau_{v} \sqrt{r^{2}+1} A_{v}\left(\tau_{v} \sqrt{r^{2}+1} \mp 0 i, r\right) h \\
& =\tau_{v} \sqrt{r^{2}+1} \int_{S} \Phi_{v}^{ \pm}(x, r, \omega) h(\omega) d \omega
\end{aligned}
$$

Thus the proof is complete.

Q.E.D.

\section{§5. Orthogonality Eigenfunctions}

Theorem 5.1. The image of the patially isometric operator $Z_{v}^{ \pm}$ on $\mathscr{L}^{2}$ coincides with $\psi_{v} \mathscr{L}^{2}$. 
Proof. We shall give the proof for $Z_{p}^{+}$alone (the other operators can be treated similarly). We know in Theorem 4.4 that the range of $Z_{p}^{+}$is contained in $\psi_{p} \mathscr{L}^{2}$. To prove that $Z_{p}^{+}$maps onto $\psi_{p} \mathscr{L}^{2}$, it suffices to verify

$$
g=Z_{p}^{+}\left(Z_{p}^{+}\right)^{*} g
$$

for every $g \in \psi_{p} \mathscr{L}^{2}$. If we assume the contrary to (5.1), there would be $g \in \psi_{p} \mathscr{L}^{2}$ such that

$$
h=g-Z_{p}^{+}\left(Z_{p}^{+}\right)^{*} g \neq 0
$$

Then let us put

$$
s_{n}=\left(Z_{p}^{+}\right)^{*} h_{n}, \quad h_{n}(x)=\left\{\begin{array}{cl}
h(x), & 1 / n<|x|<n \\
0, & \text { otherwise }
\end{array}\right.
$$

Since $h_{n}$ tends to $h$ in $\mathscr{L}^{2}$ as $n \rightarrow \infty$, we have from Theorem 4.4

$$
\begin{aligned}
s_{n}=\left(Z_{p}^{+}\right)^{*} h_{n} \longrightarrow\left(Z_{p}^{+}\right)^{*} h & =\left(Z_{p}^{+}\right)^{*} g-\left(Z_{p}^{+}\right)^{*} Z_{p}^{+}\left(Z_{p}^{+}\right)^{*} g \\
& =\left(Z_{p}^{+}\right)^{*} g-\left(Z_{p}^{+}\right)^{*} g=0 .
\end{aligned}
$$

Now we shall consider the following integral

$$
\begin{aligned}
S_{n}(z) & =(2 \pi)^{-3 / 2} \int_{0}^{+\infty} F_{p}^{+}(r) h_{n}(r \cdot) \frac{r^{2}}{\sqrt{r^{2}+1}-z} d r \\
& =(2 \pi)^{-3 / 2} \int_{1 / n}^{n} F_{p}^{+}(r) h(r \cdot) \frac{r^{2}}{\sqrt{r^{2}+1}-z} d r \in \mathscr{H}_{-t}^{1},
\end{aligned}
$$

where $\operatorname{Im} z \neq 0 . \quad S_{n}(z)$ satisfies

$$
\left(L_{0}+Q(x)-z\right) S_{n}(z)=S_{n}
$$

Indeed, it follows from Corollary 3.4 that

$$
\begin{aligned}
& \left((2 \pi)^{-3 / 2} \int_{1 / n}^{n} F_{p}^{+}(r) h(r \cdot) \frac{r^{2}}{\sqrt{r^{2}+1}-z} d r,\left(L_{0}+Q-\bar{z}\right) \varphi\right) \\
& \quad=(2 \pi)^{-3 / 2} \int_{1 / n}^{n}\left(\left(L_{0}+Q-z\right) F_{p}^{+}(r) h(r \cdot), \varphi\right) \frac{r^{2}}{\sqrt{r^{2}+1}-z} d r
\end{aligned}
$$




$$
=(2 \pi)^{-3 / 2} \int_{1 / n}^{n}\left(F_{p}^{+}(r) h(r \cdot), \varphi\right) r^{2} d r=\left(s_{n}, \varphi\right)
$$

for $\varphi \in \mathscr{C}_{0}^{\infty}$. Proposition 2.3 and (5.4) yield

$$
\left(L_{0}-z\right) S_{n}(z)=-Q S_{n}(z)+s_{n} \in \mathscr{L}^{2},
$$

which means that $S_{n}(z) \in \mathscr{H}^{1}$ and $S_{n}(z)=R(z) s_{n}$. We notice here that $S_{n}(z)$ converges to 0 in $\mathscr{L}^{2}$ as $n \rightarrow \infty$, because of (5.3). Let us take an arbitrary compact set $D$ in $\mathbb{R}^{3}-\Sigma$. Then we obtain from the above fact

$$
\lim _{n \rightarrow \infty} \int_{D} S_{n}(z) d x=0
$$

On the other hand we have

$$
\int_{D} S_{n}(z) d x=(2 \pi)^{-3 / 2} \int_{1 / n}^{n} \frac{r^{2}}{\sqrt{r^{2}+1}-z} d r \int_{D} F_{p}^{+}(r) h(r \cdot) d x
$$

(4.16) in Theorem 4.5 and (4.25) in its proof shows that

$$
\begin{aligned}
& \left(\int_{D} F_{p}^{+}(r) h(r \cdot) d x\right)_{j}=\left(F_{p}^{+}(r) h(r \cdot), \chi_{j}\right)_{\mathscr{L}^{2}} \\
& \quad=(2 \pi)^{3 / 2} \int_{S}<h(r \omega),\left(Z_{p}^{+} \chi_{j}\right)(r \omega)>d \omega \quad(j=1,2,3,4),
\end{aligned}
$$

where $\chi_{j}$ is a vector-valued function such that the $j$-th component is a characteristic function of $D$ and the other components equal 0 identical1y. This implies that

$$
\int_{0}^{+\infty}\left|\int_{D} F_{p}^{+}(r) h(r \cdot) d x\right| r^{2} d r<+\infty
$$

Thus we can take the limit of (5.6) as $n \rightarrow \infty$, and obtain

$$
\int_{0}^{+\infty} \frac{r^{2}}{\sqrt{r^{2}+1}-z} d r \int_{D} F_{p}^{+}(r) h(r \cdot) d x=0
$$

for $\operatorname{Im} z \neq 0$. Putting $z=\lambda \pm i \varepsilon$ into (5.7), integrating over $\left[\alpha^{2}+1, \beta^{2}+1\right]$ with respect to $\lambda(0<\alpha<\beta)$ and making $\varepsilon \downarrow 0$, we have, from Proposition 4.3, Fubini's theorem and Lebesgue's convergence theorem, 


$$
\begin{aligned}
0 & =\lim _{\varepsilon \downarrow 0} \int_{\sqrt{\alpha^{2}+1}}^{\sqrt{\beta^{2}+1}} d \lambda \int_{0}^{+\infty} \frac{2 i \varepsilon}{\left(\sqrt{r^{2}+1}-\lambda\right)^{2}+\varepsilon^{2}} r^{2} d r \int_{D} F_{p}^{+}(r) h(r \cdot) d x \\
& =\int_{0}^{+\infty}\left(\lim _{\varepsilon \downarrow 0} \int_{\sqrt{\alpha^{2}+1}}^{\sqrt{\beta^{2}+1}} \frac{2 i \varepsilon}{\left(\sqrt{r^{2}+1}-\lambda\right)^{2}+\varepsilon^{2}} d \lambda\right) r^{2} d r \int_{D} F_{p}^{+}(r) h(r \cdot) d x \\
& =2 \pi i \int_{\alpha}^{\beta} r^{2} d r \int_{D} F_{p}^{+}(r) h(r \cdot) d x .
\end{aligned}
$$

Hence we obtain

$$
F_{p}^{+}(r) h(r \cdot)=A_{p}\left(\sqrt{r^{2}+1}-0 i, r\right) h(r \cdot)=0
$$

for almost every $r>0$. Now we define

$$
u(x, r)=\int_{S} \Phi_{p}^{0}(x, r \omega) h(r \omega) d \omega
$$

which belongs to $\mathscr{H}_{-t}^{1}$ for a.e. $r>0$ (a.e. $=$ almost every) in view of Proposition 3.2. Then $u(x, r)$ satisfies

$$
L_{0} u=\sqrt{r^{2}+1} u
$$

for a.e. $r>0$. On the other hand it follows from (5.8) and the definition of $A_{p}$ that

$$
u=R^{-}\left(\sqrt{r^{2}+1}\right) Q u
$$

that is, $u(x, r)$ is the limit of

$$
u_{\varepsilon}(r)=R\left(\sqrt{r^{2}+1}-i \varepsilon\right) Q u
$$

as $\varepsilon \downarrow 0$ in the topology of $\mathscr{H}_{-t}^{1} . u_{\varepsilon}(r)$ fulfills, from the above definition,

$$
L_{0} u_{\varepsilon}-\left(\sqrt{r^{2}+1}-i \varepsilon\right) u_{\varepsilon}=Q\left(u-u_{\varepsilon}\right)
$$

and, as a result of Proposition 2.3,

$$
Q\left(u-u_{\varepsilon}\right) \longrightarrow 0 \quad \text { in } \quad \mathscr{L}_{t}^{2} \quad(\varepsilon \downarrow 0) .
$$

Then we can prove $u(\cdot, r) \in \mathscr{L}^{2}$ for $r>0$ by the same argument as appears in the proof of Theorem 4.1 of [15]. In view of (5.9) we see 
$u(x, r)=0$ for $x \in \mathbb{R}^{3}$ and a.e. $r>0$. Thus we obtain from (1.3) and $h=\psi_{p} h$

$$
\begin{aligned}
h(x) & =(2 \pi)^{-3 / 2} \text { li.m. } \int\left(\Phi_{p}^{0}(x, \xi)+\Phi_{n}^{0}(x, \xi)\right) h(\xi) d \xi \\
& =(2 \pi)^{-3 / 2} \text { li.m. } \int \Phi_{p}^{0}(x, \xi) h(\xi) d \xi \\
& =(2 \pi)^{-3 / 2} 1 \cdot \mathrm{i} \cdot \mathrm{m} \cdot \int_{0}^{+\infty} u(x, r) r^{2} d r=0
\end{aligned}
$$

which contradicts our hypothesis (5.2) $(h \neq 0)$. (The above proof is along the line of Ikebe [8].)

Q.E.D.

Theorem 5.2. $f \in \mathscr{L}^{2}$ belongs to the domain $D(H)$ if and only if $\sqrt{|\xi|^{2}+1}\left(Z_{v}^{ \pm} f\right)(\xi) \in \mathscr{L}^{2}(v=p$ and $n)$. For $f \in D(H)$

$$
\begin{array}{r}
H f=\left(Z_{p}^{ \pm}\right)^{*}\left\{\sqrt{|\xi|^{2}+1}\left(Z_{p}^{ \pm} f\right)(\xi)\right\}+\left(Z_{n}^{ \pm}\right)^{*}\left\{-\sqrt{|\xi|^{2}+1}\left(Z_{n}^{ \pm} f\right)(\xi)\right\} \\
+\sum_{j} \mu_{j}\left(f, \varphi_{j}\right) \varphi_{j},
\end{array}
$$

$$
\left(Z_{v}^{ \pm} H f\right)(\xi)=\tau_{v} \sqrt{|\xi|^{2}+1}\left(Z_{v}^{ \pm} f\right)(\xi)
$$

Proof. An application of (4.2) and Theorem 4.4 gives

$$
((E(\beta)-E(\alpha)) f, f)=\int_{\alpha \leqq \sqrt{r^{2}+1} \leqq \beta}\left\|\left(Z_{p}^{ \pm} f\right)(r \cdot)\right\|_{\mathscr{L}^{2}(S)}^{2} r^{2} d r(1<\alpha<\beta<+\infty)
$$

and, therefore,

$$
\frac{d}{d \mu}\|E(\mu) f\|^{2}=\mu\left(\mu^{2}-1\right)^{3 / 2}\left\|\left(Z_{p}^{ \pm} f\right)\left(\sqrt{\mu^{2}-1}\right)\right\|_{\mathscr{L}^{2}(S)}^{2}
$$

for $\mu>1$ and $f \in \mathscr{L}^{2}$. The above fact (5.12) implies

$$
\|H f\|^{2} \geqq \int_{1}^{+\infty} \mu^{2} d\|E(\mu) f\|^{2}=\int_{\mathbb{R}^{3}}\left(|\xi|^{2}+1\right)\left|\left(Z_{p}^{ \pm} f\right)(\xi)\right|^{2} d \xi
$$

for $f \in D(H)$. Therefore it turns out that

$$
\sqrt{|\xi|^{2}+1}\left(Z_{p}^{ \pm} f\right)(\xi) \in \mathscr{L}^{2}
$$

for $f \in D(H)$. Similarly we have 


$$
\sqrt{|\xi|^{2}+1}\left(Z_{n}^{ \pm} f\right)(\xi) \in \mathscr{L}^{2}
$$

for $f \in D(H)$. Thus the "only if" part of our first assertion is proved. Before proving the "if" part, we show (5.10) and (5.11) for $f \in D(H)$. It follows from a calculation similar to (5.13) that

$$
((I-E(1)) H f, g)=\int_{\mathbb{R}^{3}} \sqrt{|\xi|^{2}+1}<Z_{p}^{ \pm} f, Z_{p}^{ \pm} g>d \xi
$$

for $f \in D(H)$ and $g \in \mathscr{L}^{2}$ 。 Noting that $\sqrt{|\xi|^{2}+1}\left(Z_{p}^{ \pm} f\right)(\xi) \in \mathscr{L}^{2}$ for $f \in D(H)$ we have

$$
(I-E(1)) H f=\left(Z_{p}^{ \pm}\right)^{*}\left\{\sqrt{|\bar{\xi}|^{2}+1}\left(Z_{p}^{ \pm} f\right)(\xi)\right\}
$$

for $f \in D(H)$. In the same manner we obtain

$$
E(-1-0) H f=\left(Z_{n}^{ \pm}\right)^{*}\left\{-\sqrt{|\xi|^{2}+1}\left(Z_{n}^{ \pm} f\right)(\xi)\right\}
$$

for $f \in D(H)$. According to the expansion theorem for Fourier series we have

$$
\begin{aligned}
(E(1)-E(-1-0)) H f & =\sum_{j}\left(H f, \varphi_{j}\right) \varphi_{j}=\sum_{j}\left(f, H \varphi_{j}\right) \varphi_{j} \\
& =\sum_{j} \mu_{j}\left(f, \varphi_{j}\right) \varphi_{j} .
\end{aligned}
$$

for $f \in D(H)$. Thus (5.10) follows from (5.14), (5.15) and (5.16). Since $Z_{\bar{p}}^{ \pm}$and $Z_{n}^{ \pm}$are partially isometric operators with the initial sets ( $I$ $-E(1)) \mathscr{L}^{2}$ and $E(-1-0) \mathscr{L}^{2}$, respectively, as a result of Theorem 4.4, we have

$$
\begin{aligned}
& Z_{p}^{ \pm}\left(Z_{n}^{ \pm}\right)^{*}=Z_{n}^{ \pm}\left(Z_{p}^{ \pm}\right)^{*}=0 \\
& Z_{p}^{ \pm} \varphi_{j}=Z_{n}^{ \pm} \varphi_{j}=0
\end{aligned}
$$

Therefore (5.11) is verified from (5.1) and (5.10) as

$$
Z_{v}^{ \pm} H f=Z_{v}^{ \pm}\left(Z_{v}^{ \pm}\right)^{*}\left\{\tau_{v} \sqrt{|\xi|^{2}+1}\left(Z_{v}^{ \pm} f\right)(\xi)\right\}=\tau_{v} \sqrt{|\xi|^{2}+1}\left(Z_{v}^{ \pm} f\right)(\xi) .
$$

Finally we prove that $f \in \mathscr{L}^{2}$ is contained in $D(H)$ if $\sqrt{|\xi|^{2}+1}\left(Z_{v}^{ \pm} f\right)(\xi)$ $\in \mathscr{L}^{2}(v=p, n)$. Let

$$
\begin{aligned}
\tilde{H} f=\left(Z_{p}^{ \pm}\right)^{*}\left\{\sqrt{|\xi|^{2}+1}\left(Z_{p}^{ \pm} f\right)(\xi)\right\}+\left(Z_{n}^{ \pm}\right)^{*}\left\{-\sqrt{|\xi|^{2}+1}\left(Z_{n}^{ \pm} f\right)(\xi)\right\} \\
+\sum_{j} \mu_{j}\left(f, \varphi_{j}\right) \varphi_{j}
\end{aligned}
$$


for $f \in \mathscr{L}^{2}$ such that $\sqrt{|\xi|^{2}+1}\left(Z_{v}^{ \pm} f\right)(\xi) \in \mathscr{L}^{2}(v=p, n)$. Since

$$
\left\|\sum_{j} \mu_{j}\left(f, \varphi_{j}\right) \varphi_{j}\right\|^{2} \leqq \sum_{j}\left|\left(f, \varphi_{j}\right)\right|^{2} \leqq\|f\|^{2}
$$

for $f \in \mathscr{L}^{2}$ (because of $\left|\mu_{j}\right| \leqq 1$ ), the third term in the above definition (5.17) makes sense in $\mathscr{L}^{2}$. From the definition, $\tilde{H}$ is obviously a symmetric operator in $\mathscr{L}^{2}$. Moreover, the domain $D(\tilde{H})$ includes $D(H)$, as has been seen at the beginning of our proof. Therefore, $\tilde{H}$ is a symmetric extension of the selfadjoint operator $H$. Hence $H$ must coincide with $H$ (see Yosida [16], p. 350).

Q.E.D.

Remark 5.3. For every real $t$

$$
\begin{array}{r}
e^{i t H} f=\left(Z_{p}^{ \pm}\right)^{*}\left\{e^{i t \sqrt{|\xi|^{2}+1}}\left(Z_{p}^{ \pm} f\right)(\xi)\right\}+\left(Z_{n}^{ \pm}\right)\left\{e^{-i t \sqrt{|\xi|^{2}+1}}\left(Z_{n}^{ \pm} f\right)(\xi)\right\} \\
+\sum_{j} e^{i t \mu J}\left(f, \varphi_{j}\right) \varphi_{j},
\end{array}
$$

for $f \in \mathscr{L}^{2}$, where $e^{i t H}=\int e^{i t \lambda} d E(\lambda)$.

The proof of the above remark can be given along the same line as the proof of Theorem 5.2.

\section{§6. Wave and Scattering Operators}

The wave operators are defined as

$$
\Omega_{ \pm}=\mathrm{S}-\lim _{t \rightarrow \pm \infty} e^{i t H^{-i t H_{0}}}
$$

when the right-hand strong limits exist. Then $S=\Omega_{+}^{*} \Omega_{-}$is called the scattering operator. According to Kato [9], Theorem X-3.2, the wave operator, if it exists, is isometric with the final set included in $(I-E(1)$ $+E(-1-0)) \mathscr{L}^{2}$.

The following proposition is proved by Prosser [11].

Proposition 6.1. Let $Q(x)$ be a $4 \times 4$ matrix-valued function such that its components are locally in $L^{2}\left(\mathbb{R}^{3}\right)$, and

$$
|Q(x)| \leqq \frac{\text { const. }}{(1+|x|)^{1+h}} \quad\left(|x| \geqq R_{0}, h>0\right) .
$$


Then if $f(x)$ is infinitely differentiable with compact support,

$$
\int_{-\infty}^{+\infty}\left\|Q e^{-i t H_{0}} f\right\| d t<+\infty
$$

In consequence of the above proposition and Theorem X-3.7 in Kato [9], the wave operators $\Omega_{ \pm}$exist for our Dirac operator satisfying the condition (A) in $\S 2$. Our goal in this section is to show

$$
\Omega_{ \pm} f=\left(Z_{p}^{ \pm}\right)^{*} Z_{p}^{0} f+\left(Z_{n}^{ \pm}\right)^{*} Z_{n}^{0} f
$$

for every $f \in \mathscr{L}^{2}$, and that the scattering operator is unitary. To this end we prepare several lemmas.

Lemma 6.2. Let us define

$$
W_{ \pm} f=\left(Z_{p}^{ \pm}\right)^{*} Z_{p}^{0} f+\left(Z_{n}^{ \pm}\right)^{*} Z_{n}^{0} f
$$

for $f \in \mathscr{L}^{2}$. Then $W_{ \pm}$is an isometric operator from $\mathscr{L}^{2}$ onto (I-E(1) $+E(-1-0)) \mathscr{L}^{2}$, satisfying

$$
\begin{aligned}
& Z_{v}^{ \pm}=Z_{v}^{0} W_{ \pm}^{*} \quad(v=p, n) \\
& W_{ \pm}^{*} H=H_{0} W_{ \pm}^{*} \\
& W_{ \pm}^{*} e^{i t H}=e^{i t H_{0}} W_{ \pm}^{*}, \quad-\infty<t<+\infty .
\end{aligned}
$$

(The isometric operator $W_{ \pm}$is called the stationary wave operator.)

Proof. Since $Z_{p}^{ \pm}\left(Z_{n}^{ \pm}\right)$are partially isometric operators with the initial set $(I-E(1)) \mathscr{L}^{2}\left(E(-1-0) \mathscr{L}^{2}\right)$ and the final set $\psi_{p} \mathscr{L}^{2}\left(\psi_{n} \mathscr{L}^{2}\right)$,

$W_{ \pm}$are isometric operators, that is,

$$
\begin{aligned}
\left\|W_{ \pm} f\right\|^{2} & =\left\|\left(Z_{p}^{ \pm}\right)^{*} Z_{p}^{0} f\right\|^{2}+\left\|\left(Z_{n}^{ \pm}\right)^{*} Z_{n}^{0} f\right\|^{2} \\
& =\left\|Z_{p}^{0} f\right\|^{2}+\left\|Z_{n}^{0} f\right\|^{2}=\|f\|^{2}
\end{aligned}
$$

Furthermore we have

$$
\begin{aligned}
& W_{ \pm}^{*}=\left(Z_{p}^{0}\right)^{*} Z_{p}^{ \pm}+\left(Z_{n}^{0}\right)^{*} Z_{n}^{ \pm} \\
& Z_{v}^{0} W_{ \pm}^{*}=Z_{v}^{0}\left(Z_{p}^{0}\right)^{*} Z_{p}^{ \pm}+Z_{v}^{0}\left(Z_{n}^{0}\right)^{*} Z_{n}^{ \pm}=Z_{v}^{ \pm}
\end{aligned}
$$


In order to prove (6.3) we make use of Theorem 5.2 to get

$$
\begin{aligned}
W_{ \pm}^{*} H f & =\left(Z_{p}^{0}\right)^{*} Z_{p}^{ \pm} H f+\left(Z_{n}^{0}\right) * Z_{n}^{ \pm} H f \\
& =\left(Z_{p}^{0}\right)^{*}\left\{\sqrt{|\xi|^{2}+1}\left(Z_{p}^{ \pm} f\right)(\xi)\right\}-\left(Z_{n}^{0}\right)^{*}\left\{\sqrt{|\xi|^{2}+1}\left(Z_{n}^{ \pm} f\right)(\xi)\right\} \\
& =\left(Z_{p}^{0}\right) *\left\{\sqrt{|\xi|^{2}+1}\left(Z_{p}^{0} W_{ \pm}^{*} f\right)(\xi)\right\}-\left(Z_{n}^{0}\right)^{*}\left\{\sqrt{|\xi|^{2}+1}\left(Z_{n}^{0} W_{+} f\right)(\xi)\right\} \\
& =H_{0} W_{ \pm}^{*} f
\end{aligned}
$$

for $f \in D(H)$, which gives $W_{ \pm}^{*} H \subset H_{0} W_{ \pm}^{*}$. The other inclusion is obtained by an application of Theorem 5.2 again. If $W_{ \pm}^{*} f$ is contained in the domain $D\left(H_{0}\right)=\mathscr{H}^{1}\left(\mathbb{R}^{3}\right)$, then

$$
\sqrt{|\xi|^{2}+1}\left(Z_{v}^{ \pm} f\right)(\xi)=\sqrt{|\xi|^{2}+1} Z_{v}^{0} W_{ \pm}^{*} f \in \mathscr{L}^{2} \quad(v=p, n),
$$

which implies $f \in D(H)$ by means of Theorem 5.2. Hence we have $D\left(H_{0} W_{ \pm}^{*}\right) \subset D\left(W_{ \pm}^{*} H\right)$. Thus (6.3) is proved, and (6.4) is a well-known consequence of (6.3) (see the proof of Theorem X-3.2 in Kato [9]). Q.E.D.

Lemma 6.3. Let $f$ belong to $D\left(H_{0}\right)$. Then

$$
\frac{d}{d t} Q e^{i t\left(H_{0}-z\right)} R_{0}(z) f=i Q e^{i t\left(H_{0}-z\right)} f \quad \text { in } \mathscr{L}_{t}^{2}
$$

for $\operatorname{Im} z \neq 0$ and $-\infty<t<+\infty$.

The proof of the above lemma is given by straightforward calculations in view of Proposition 2.3.

Lemma 6.4. For any real $\lambda$ such that $|\lambda|>1$

$$
R^{ \pm}(\lambda)=R_{0}^{ \pm}(\lambda)-R^{ \pm}(\lambda) Q R_{0}^{ \pm}(\lambda)
$$

Proof. The lemma is almost trivial from Propositions 2.2 and 2.3 and the (second) resolvent equation

$$
R(z)=R_{0}(z)-R(z) Q R_{0}(z)
$$

for non-real $z$.

Q.E.D. 
The following lemma is evident from Lebesgue's convergence theorem.

Lemma 6.5. Let $\varphi(s)$ be an integrable function on $[0,+\infty)$. Then

$$
\lim _{t \rightarrow+\infty} \int_{0}^{t} \varphi(s) d s=\lim _{\varepsilon \downarrow 0} \int_{0}^{+\infty} e^{-\varepsilon s} \varphi(s) d s
$$

Theorem 6.6. $W_{ \pm}$(defined in Lemma 6.2) coincides with the wave operator $\Omega_{ \pm}$, i.e.,

$$
W_{ \pm}=\mathrm{s}-\lim _{t \rightarrow \pm \infty} e^{i t H} e^{-i t H_{0}}
$$

Proof. It is enough to show $W_{ \pm}^{*} \Omega_{ \pm}=I$, because then, by Lemma 6.2 ,

$$
W_{ \pm}=W_{ \pm} W_{ \pm}^{*} \Omega_{ \pm}=(I-E(1)+E(-1-0)) \Omega_{ \pm}=\Omega_{ \pm} \text {. }
$$

Since

$$
\frac{d}{d t} e^{i t H} e^{-i t H_{0}} f=i e^{i t H}\left(H-H_{0}\right) e^{-i t H_{0}} f=i e^{i t H} Q e^{-i t H_{0}}
$$

in $\mathscr{L}^{2}$ for every $f \in D(H)=D\left(H_{0}\right)$, we have

$$
\left(W_{+}^{*} e^{i t H} e^{-i t I I \circ} f, g\right)-\left(W_{+}^{*} f, g\right)=i \int_{0}^{t}\left(W_{+}^{*} e^{i s H} Q e^{-i s H_{0}} f, g\right) d s
$$

for $f \in D(H)$ and $g \in \mathscr{L}^{2}$. Lemma 6.2, (6.4), yields

$$
(6.5)=i \int_{0}^{t}\left(e^{i s H_{0}} W_{+}^{*} Q e^{-i s H_{0}} f, g\right) d s .
$$

By Remark 5.3 and (6.2) in Lemma 6.2 the above integrand can be changed into

$$
\begin{aligned}
& \left(e^{i s H_{0}} W_{+}^{*} Q e^{-i s H_{0}} f, g\right) \\
= & \sum_{v=p, n}\left(\left(Z_{v}^{0}\right)^{*}\left\{e^{i \tau_{v} s \sqrt{|\xi|^{2}+1}}\left(Z_{v}^{0} W_{+}^{*} Q e^{-i s H_{0}} f\right)(\xi)\right\}, g\right)_{\mathscr{L}^{2}} \\
= & \sum_{v=p, n} \int_{\mathbf{R}^{3}}<e^{i \tau_{v} s \sqrt{|\xi|^{2}+1}}\left(Z_{v}^{+} Q e^{-i s H_{0}} f\right)(\xi),\left(Z_{v}^{0} g\right)(\xi)>d \xi .
\end{aligned}
$$


Hence we have

$$
\begin{aligned}
& \left(W_{+}^{*} e^{i t H} e^{-i t H_{0}} f, g\right)-\left(W_{+}^{*} f, g\right) \\
= & i \int_{0}^{t} d s \sum_{v=p, n} \int_{\mathbb{R}^{3}}<e^{i \tau_{v} s \sqrt{|\xi|^{2}+1}}\left(Z_{v}^{+} Q e^{-i H_{0}} f\right)(\xi),\left(Z_{v}^{0} g\right)(\xi)>d \xi .
\end{aligned}
$$

Take the limit of $(6.6)$ as $t \rightarrow \infty$. Then we see from Lemma 6.5 that

$$
\begin{aligned}
& \left(W_{+}^{*} \Omega_{+} f, g\right)-\left(W_{+}^{*} f, g\right) \\
= & \lim _{\varepsilon \downarrow 0} i \int_{0}^{+\infty} e^{-\varepsilon s} d s \sum_{v} \int_{\mathbb{R}^{3}}<e^{i \tau_{v} s \sqrt{|\xi|^{2}+1}}\left(Z_{v}^{+} Q e^{-i s H_{0}} f\right)(\xi),\left(Z_{v}^{0} g\right)(\xi)>d \xi \\
= & \lim _{\varepsilon \downarrow 0} i \sum_{v} \int_{0}^{+\infty} r^{2} d r \int_{0}^{+\infty}\left(Z_{v}^{+} Q e^{-i s\left(H_{0}-\tau_{v} \sqrt{r^{2}+1}-i \varepsilon\right)} f,\left(Z_{v}^{0} g\right)\left(r_{\bullet}\right)\right)_{\mathscr{L}^{2}(S)} d s,
\end{aligned}
$$

where we have used Fubini's theorem. Let us recall the definition (4.9) of $Z_{v}^{+}$, and note $Q e^{-i s H_{0}} f \in \mathscr{L}_{t}^{2}$ for $f \in D\left(H_{0}\right)$, because of $e^{-i s H_{0}} f$ $\in D\left(H_{0}\right)$ and Proposition 2.3. Then we have

$$
\begin{array}{r}
\left(W_{+}^{*} \Omega_{+} f, g\right)-\left(W_{+}^{*} f, g\right) \\
=\lim _{\varepsilon \downarrow 0} i(2 \pi)^{-3 / 2} \sum_{v} \int_{0}^{+\infty} r^{2} d r \int_{0}^{+\infty}\left(\left(F_{v}^{+}(r)\right)^{*} Q e^{-i s\left(H_{0}-\tau_{\nu} \sqrt{r^{2}+1}-i \varepsilon\right)} f,\right. \\
\left.\left(Z_{v}^{0} g\right)(r \cdot)\right)_{\mathscr{L} 2(S)} d s
\end{array}
$$

for $f \in D\left(H_{0}\right)$ and $g \in \mathscr{L}^{2}$. Lemma 6.3 yields that

$$
\begin{aligned}
& \left(W_{+}^{*} \Omega_{+} f, g\right)-\left(W_{+}^{*} f, g\right) \\
& =-\lim _{\varepsilon \downarrow 0}(2 \pi)^{-3 / 2} \sum_{v} \int_{0}^{+\infty} r^{2} d r \int_{0}^{+\infty} \frac{d}{d s}\left(\left(F_{v}^{+}(r)\right)^{*} Q\right. \\
& \left.e^{-i s\left(H_{0}-\tau_{v} \sqrt{r^{2}+1}-i \varepsilon\right)} R_{0}\left(\tau_{v} \sqrt{r^{2}+1}+i \varepsilon\right) f,\left(Z_{v}^{0} g\right)\left(r^{\circ}\right)\right)_{\mathscr{L}^{2}(S)} d s \\
& =\lim _{\varepsilon \downarrow 0}(2 \pi)^{-3 / 2} \sum_{v} \int_{0}^{+\infty}\left(\left(F_{v}^{+}(r)\right)^{*} Q R_{0}\left(\tau_{v} \sqrt{r^{2}+1}+i \varepsilon\right) f,\left(Z_{v}^{0} g\right)(r \cdot)\right)_{\mathscr{L}^{2}(S)} r^{2} d r .
\end{aligned}
$$

Since

$$
\begin{gathered}
\left\|\left(F_{v}^{+}(r)\right)^{*} Q R_{0}\left(\tau_{v} \sqrt{r^{2}+1}+i \varepsilon\right) f\right\|_{\mathscr{L}^{2}(S)} \leqq C(a, b)\|f\|_{\mathscr{L}_{t}^{2}} \\
(0<a \leqq r \leqq b<+\infty, 0<\varepsilon \leqq 1)
\end{gathered}
$$


by virtue of Lemma 3.8 , we obtain

$$
\begin{aligned}
& \left(W_{+}^{*} \Omega_{+} f, g\right)-\left(W_{+}^{*} f, g\right) \\
& \quad=(2 \pi)^{-3 / 2} \sum_{v} \int_{0}^{+\infty}\left(\left(F_{v}^{+}(r)\right)^{*} Q R_{0}^{+}\left(\tau_{v} \sqrt{r^{2}+1}\right) f,\left(Z_{v}^{0} g\right)(r \cdot)\right)_{\mathscr{L}^{2}(S)} r^{2} d r
\end{aligned}
$$

for $f \in \mathscr{C}_{0}^{\infty} \subset D\left(H_{0}\right) \cap \mathscr{L}_{t}^{2}$ and $\hat{g} \in \mathscr{C}_{0}^{\infty}\left(\mathbb{R}^{3}-\{0\}\right)$. It follows from the definition of $W_{+}$that

$$
\begin{aligned}
\left(W_{+}^{*} f, g\right) & =\left(\sum_{v}\left(Z_{v}^{0}\right)^{*} Z_{v}^{+} f, g\right)=\sum_{v}\left(Z_{v}^{+} f, Z_{v}^{0} g\right) \\
& =(2 \pi)^{-3 / 2} \sum_{v} \int_{0}^{+\infty}\left(\left(F_{v}^{+}(r)\right)^{*} f,\left(Z_{v}^{0} g\right)(r \cdot)\right)_{\mathscr{L}^{2}(S)} r^{2} d r
\end{aligned}
$$

for $f \in \mathscr{L}_{t}^{2}$ and $g \in \mathscr{L}^{2} . \quad(6.7)$ and (6.8) are combined to show

$$
\begin{aligned}
\left(W_{+}^{*} \Omega_{+} f, g\right)=(2 \pi)^{-3 / 2} \sum_{v} \int_{0}^{+\infty}\left(\left(F_{v}^{+}(r)\right) *(f+\right. & Q R_{0}^{+}\left(\tau_{v} \sqrt{r^{2}+1}\right) f, \\
& \left.\left(Z_{v}^{0} g\right)(r \cdot)\right)_{\mathscr{L}^{2}(S)} r^{2} d r .
\end{aligned}
$$

Corollary 3.9, Definition 4.1 and Lemma 6.4 give that

$$
\begin{aligned}
(2 \pi)^{-3 / 2}\left(\left(F_{v}^{+}(r)\right)^{*}\left(f+Q R_{0}^{+}\left(\tau_{v} \sqrt{r^{2}+1}\right) f\right)\right. \\
=\psi_{v}(r \cdot)\left\{(\mathscr{F} f)(r \cdot)+\left(\mathscr{F} Q R_{0}^{+}\left(\tau_{v} \sqrt{r^{2}+1}\right) f\right)(r \cdot)\right\} \\
\quad-\psi_{v}(r \cdot)\left(\mathscr{F} Q R^{+}\left(\tau_{v} \sqrt{r^{2}+1}\right)\left(f+Q R_{0}^{+}\left(\tau_{v} \sqrt{r^{2}+1}\right) f\right)\right)(r \cdot) \\
=\psi_{v}(r \cdot)(\mathscr{F} f)(r \cdot),
\end{aligned}
$$

where $\mathscr{F}$ denotes the Fourier transform. Thus we have finally

$$
\begin{aligned}
\left(W_{+}^{*} \Omega_{+} f, g\right) & =\sum_{\nu} \int_{0}^{+\infty}\left(\psi_{v}(r \cdot) \hat{f}(r \cdot),\left(Z_{v}^{0} g\right)(r \cdot)\right)_{\mathscr{L}^{2}(S)} r^{2} d r \\
& =(\hat{f}, \hat{g})=(f, g)
\end{aligned}
$$

for $f \in \mathscr{C}_{0}^{\infty}$ and $\hat{g} \in \mathscr{C}_{0}^{\infty}\left(\mathbf{R}^{3}-\{0\}\right)$. As $\mathscr{C}_{0}^{\infty}$ and $\mathscr{C}_{0}^{\infty}\left(\mathbf{R}^{3}-\{0\}\right)$ are dense in $\mathscr{L}^{2}$, we have $W_{+}^{*} \Omega_{+}=I$. A similar argument applies to show $W_{-}^{*} \Omega_{-}$ $=I$. Thus we have completed the proof. $\quad$ Q.E.D.

Theorem 6.7. The scattering operator is unitary. 
Proof. Since the image of the isometric operator $W_{-}\left(W_{+}\right)$is ( $I$ $-E(1)+E(-1-0)) \mathscr{L}^{2}$ and the adjoint operator $W_{+}^{*}$ (of the isometric operator $\left.W_{+}\right)$maps $(I-E(1)+E(-1-0)) \mathscr{L}^{2}$ onto $\mathscr{L}^{2}$ in consequence of Lemma 6.2, the scattering operator

$$
S=\Omega_{+}^{*} \Omega_{-}=W_{+}^{*} W_{-}
$$

is unitary.

Q.E.D.

In concluding this paper we give the final remark.

Remark 6.8. One of our assumptions on $Q(x)$ (in $\S 2$ )

$$
\int_{|x| \leqq R_{0}}|Q(x)|^{p} d x<+\infty
$$

for some $p>3$, can be replaced by a somewhat weaker condition

$$
\sup _{x \in B_{R_{0}+1}} \int_{|x-y| \leqq 1} \frac{|Q(y)|^{2}}{|x-y|^{1+\theta}} d y<+\infty
$$

for some $0<\theta \leqq 1$. The condition (a.3) is assumed in the present paper and $[15]$ merely to obtain

$$
\int_{B_{R_{0}}}|Q u|^{2} d x \leqq \int_{B_{R_{0}+1}}\left|L_{0} u\right|^{2} d x+C_{\varepsilon} \int_{B_{R_{0}+1}}|u(x)|^{2} d x
$$

for any $\varepsilon>0$ and $u \in \mathscr{H}^{1}\left(B_{R_{0}+1}\right)$. Evans [5] in his Lemma 7 (which came to the author's attention after the completion of [15]) proves (2.2) under the condition (a.3)'. Therefore all our assertions hold without any modifications, supposing the conditions (a.1), (a.2) and (a.3)'。

Acknowledgement. The author wishes to express his sincere gratitude to Prof. T. Ikebe and Prof. Y. Saito for their enduring encouragements and valuable instructions.

\section{References}

[1] Agmon, S., Lectures at the Oberwalfach symposium on mathematical theory of scattering (1971).

[2] Agmon, S., On kernels, eigenvalues, and eigenfunctions of operators related to 
elliptic problems, Comm. Pure Appl. Math., 18 (1965), 627-663.

[3] Eckardt, K. J., On the existence of wave operators for Dirac operators, Manuscripta Math., 11 (1974), 359-371.

[4] Eckardt, K. J., Scattering theory for Dirac operators, Math. Z., 139 (1974), 105-131.

[5] Evans, W.D., On the unique self-adjoint extension of the Dirac operator and the existence of the Green matrix, Proc. London Math. Soc., 20 (1970), 537557.

[6] Hille, E. and Phillips, R.S., Functional analysis and semi-groups, Amer. Math. Soc. Coll. Publ., 31 (1957).

[7] Ikebe, T., Eigenfunction expansions associated with the Schrödinger operators and their applications to scattering theory, Arch. Rational Mech. Anal., 5 (1960), 1-34.

[8] Ikebe, T., Orthogonality of the eigenfunctions for the exterior problem connected with - 1, Arch. Rational Mech. Anal., 19 (1965), 71-73.

[9] Kato, T., Perturbation theory for linear operators, Springer, Berlin, (1966).

[10] Mochizuki, K., On the perturbation of the continuous spectrum of the Dirac operator, Proc. Japan Acad., 40 (1964), 707-712.

[11] Prosser, R. T., Relativistic potential scattering, J. Mathematical Phys., 4 (1963), 1048-1054.

[12] Sobolev, S. L., Applications of functional analysis in mathematical physics, Amer. Math. Soc., Providence, (1963).

[13] Thompson, M., Eigenfunction expansions and the associated scattering theory for potential perturbations of the Dirac equation, Quart. J. Math. Oxford (2), 23 (1972), 17-55.

[14] Titchmarsh, E. C., On the completeness problem for the eigenfunction formulae of relativistic quantum mechanics, Proc. Roy. Soc. Ser. A, 262 (1961), 489-502.

[15] Yamada, O., On the principle of limiting absorption for the Dirac operator, Publ. RIMS, Kyoto Univ., 8 (1973), 557-577.

[16] Yosida, K., Functional analysis, Springer, Berlin, (1968). 
